\title{
Quantum origin of the primordial fluctuation spectrum and its statistics
}

\author{
Susana Landau, ${ }^{1, *}$ Gabriel León, ${ }^{2,3, \dagger}$ and Daniel Sudarsky ${ }^{3,4, *}$ \\ ${ }^{1}$ Instituto de Física de Buenos Aires, CONICET-UBA, Ciudad Universitaria-Pab. 1, 1428 Buenos Aires, Argentina \\ ${ }^{2}$ Dipartimento di Fisica, Università di Trieste, Strada Costiera 11, 34151 Trieste, Italy \\ ${ }^{3}$ Instituto de Ciencias Nucleares, Universidad Nacional Autónoma de México, México D.F. 04510, Mexico \\ ${ }^{4}$ Instituto de Astronomía y Física del Espacio, Casilla de Correos 67, Sucursal 28, 1428 Buenos Aires, Argentina
}

(Received 3 July 2012; revised manuscript received 23 April 2013; published 26 July 2013)

\begin{abstract}
The usual account for the origin of cosmic structure during inflation is not fully satisfactory, as it lacks a physical mechanism capable of generating the inhomogeneity and anisotropy of our Universe, from an exactly homogeneous and isotropic initial state associated with the early inflationary regime. The proposal in [A. Perez, H. Sahlmann, and D. Sudarsky, Classical Quantum Gravity 23, 2317 (2006)] considers the spontaneous dynamical collapse of the wave function as a possible answer to that problem. In this work, we review briefly the difficulties facing the standard approach, as well as the answers provided by the above proposal and explore their relevance to the investigations concerning the characterization of the primordial spectrum and other statistical aspects of the cosmic microwave background and large-scale matter distribution. We will see that the new approach leads to novel ways of considering some of the relevant questions, and, in particular, to distinct characterizations of the non-Gaussianities that might have left imprints on the available data.
\end{abstract}

DOI: $10.1103 /$ PhysRevD.88.023526

PACS numbers: $98.80 . \mathrm{Cq}, 98.80 .-\mathrm{k}$

\section{INTRODUCTION}

At what point in the cosmic evolution do the actual primordial inhomogeneities arise? In other words, when does our Universe depart from the exceedingly high homogeneity and isotropy ${ }^{1}$ that is thought to result from the first stages of inflation? This is a question that one might expect should be addressed, at least, in principle, by any theory that deals with the emergence of cosmic structure. Yet in the standard inflationary account [1], which is nowadays regarded as a remarkable success, the context in which such issues can be addressed seems to be simply absent [2]. That is, within the orthodox accounts, one cannot identify the physical process responsible for the generation of those features in our Universe. In fact, according to the inflationary paradigm, from a relatively wide initial set of possibilities marking the end of the mysterious quantum gravity era, the accelerated inflationary burst leads to a homogeneous and isotropic (H\&I) Universe where the quantum fields are all characterized by the equally homogeneous and isotropic vacuum states (usually taken specifically to be the so-called Bunch-Davies vacuum). From these conditions, it is usually argued, in a rather unclear ${ }^{2}$ although strongly image-evoking manner, that the "quantum fluctuations" present in such a quantum state morph into the seeds

\footnotetext{
*slandau@df.uba.ar

gabriel.leon@nucleares.unam.mx

sudarsky@nucleares.unam.mx

${ }^{1}$ The level of inhomogeneity that might still be present at any point during inflation is expected to be of order $e^{-N}$, where $N$ is the number of e-folds of inflation occurred up to that point.

${ }^{2}$ Acknowledgments that this is an unclear aspect of the standard approach can be seen, for instance, in the book Cosmology by Weinberg [3], where the author explicitly states his view on the subject.
}

of anisotropies and inhomogeneities that characterize our late Universe. This issue is sometimes characterized as the "transition from the quantum regime to the classical regime," but we find this a bit misleading: most people would agree that there exist no distinct and separated classical and quantum regimes. The fundamental description ought to be always quantum mechanical; the so-called classical regimes are those in which certain quantities can be described to a sufficient accuracy by their classical counterparts representing the corresponding quantum expectation values. The paradigmatic example of this classical regime is provided by the coherent states of a harmonic oscillator, which correspond to minimal wave packets with expectation values of position and momentum that follow the classical equations of motion (Ehrenfest theorem). In any case, it seems clear that from a situation corresponding to a H\&I background, and quantum fields characterized by a H\&I state, one cannot end up-in the absence of something else, which in other circumstances would be identified as a measurement, but which clearly cannot be invoked in the present setting ${ }^{3}$-in a situation that is characterized, at any level, as containing actual inhomogeneities and anisotropies. It is clear that, in terms of the standard dynamics, such a transition cannot be accounted for by anything that relies just on the gravity/inflaton action, ${ }^{4}$ which is known to preserve such

\footnotetext{
${ }^{3}$ Observers and measuring apparatuses are only possible well after the H\&I has been broken, so those can hardly be part of the cause of the breakdown.

${ }^{4}$ In fact, even the interaction with other fields, controlled by the usual symmetry preserving dynamics, cannot account for the emergence of inhomogeneities and anisotropies, since, according to the inflationary paradigm, the state for all fields should correspond to a homogenous and isotropic state such as the Bunch-Davies vacuum.
} 
symmetries. Simply put, if the initial state is H\&I and the Schrödinger evolution is tied to a Hamiltonian that preserves these symmetries, the resulting state cannot be anything but a H\&I state (see Appendix A). Nonetheless, various types of arguments are often put forward in attempts to bypass the above conclusion. Most cosmologists adopt a posture that attributes to decoherence the role of explaining the emergence of inhomogeneities and anisotropies from the H\&I state. This approach faces several problems:

(i) The decoherence program is based on partitioning the degrees of freedom in two categories-The degrees of freedom of the environment and the degrees of freedom of the system of interest. In the cosmological case however, in which one cannot evoke observers or measurements, the way to do the separation of the degrees of freedom is rather ad hoc.

(ii) In order to argue that the symmetry was broken, one needs to assume that the world is suddenly represented by one of the elements appearing in the diagonal of the deciphering density matrix, and it is not clear how to argue for that.

(iii) Sometimes people evoke the many worlds interpretation of quantum theory in order to deal with the previous point but seem to ignore that, in order to do that, one needs to choose a privileged basis associated with the world splittings, and that choice, in practice, is tied to the notion of conscience, again a notion that cannot be invoked in the context at hand. Another popular posture is to rely on the consistent histories approach, ignoring the problematic issues afflicting that proposal. In particular, we should note that the usage of the formalism requires a choice of realm, a choice that in the current context seems completely arbitrary. In fact, one can make one such choice when one is led to the conclusion that the Universe is, even today, perfectly homogenous and isotropic (see Appendix D).

The extended discussion of the conceptual problems inherent to quantum theory and those associated to its application to the cosmological situation at hand have been presented in previous works by some of us and by others in Refs. [4-6]. The main message is that the problem we face is tied with the so-called measurement problem of quantum theory and that this problem becomes exacerbated in the present case, in which we are dealing with cosmology, a field in which the standard ways to address such problems are simply unavailable [7]. In this work, we reproduce all those arguments in detail, mentioning them only briefly, as the main objective of the present manuscript is to focus in the statistical aspects that emerge in this context (a slightly more detailed discussion of those is offered in Appendix D for the benefit of the reader).

We will discuss a new way of looking at those issues, based on what we consider to be a conceptually more transparent picture that relies on a modified version of the standard inflationary paradigm, which we have been advocating in previous works [4,8-11]. The basis of that proposal is to modify the standard inflationary paradigm with the incision of a modified quantum mechanics that involves the spontaneous collapse of the wave function.

We should note, however, that we cannot escape from the related problems, even if we choose to adopt a very "pragmatic position": Assume, that one chooses to ignore the shortcomings of the standard accounts [5] and accepts that, say, decoherence addresses somehow the issue at hand and that the mystery lies "only" in the question concerning the precise mechanism that lies behind the fact that, from the options exhibited in those analyses (i.e., the options displayed in the diagonal reduced-density matrix; see Appendix D), one single realization seems to be selected [12] for our Universe. In adopting such a point of view, one would be assuming that the initial symmetry has been lost (at least for practical purposes) in association with that particular "realization" or "actualization" (represented by a particular element in the density matrix). Thus, it seems clear that, for the sake of self-consistency, one should consider, when studying aspects of the inhomogeneity and anisotropies in the cosmic microwave background (CMB) we observe, the state corresponding to such realization or actualization, and not the complete vacuum state, which describes the H\&I state of affairs previous to the actualization. ${ }^{5}$ In following such views, the discussion that we are presenting in this paper would have to be taken to represent the effective description corresponding to "our perceived Universe" (in a context in which one puts together something like the many worlds interpretation, with the arguments based on decoherence). Although we definitely do not adhere to such a view for the reasons explained in Ref. [5], it is clear that when accepting a description, such as the one presented above, one would have to use the characteristics of the selected state in order to estimate the details of the inhomogeneities and anisotropies in the cosmic structure and its imprints in the CMB.

As we indicated, the purpose of this paper is to discuss the manner in which the consideration of statistical aspects of the CMB and the large-scale matter distributions should

\footnotetext{
${ }^{5}$ The reliance on a particular realization or actualization refers, of course, to the fact that, according to the standard arguments, the resulting density matrix, after becoming essentially diagonal due to decoherence, would be taken to represent an ensemble of universes, with our particular one corresponding to one of the elements occurring in the diagonal of that matrix. That state should then be considered as somehow "selected by nature" to become realized (or to be the one we perceive). If one wanted to consider the issue at a deeper level, one would have to face the question of what such actualization represents at the theoretical level and what is, if any, the physics that controls it. Alternatively, one might take the view (often referred as the many worlds interpretation) that these other universes are somehow also realized, and thus they exist in realms completely inaccessible to us. In that case, the actualization corresponds to that Universe in which we happen to exist.
} 
be modified when taking into account the modifications needed to explain the emergence of inhomogeneities and anisotropies in terms of theories incorporating something like the spontaneous collapse of the wave function. The need to rely on a different approach to study things like the non-Gaussianities in the CMB, arises, in part, due to the vastly larger space of possibilities for exotic effects, which opens in connection to the unknown dynamics of the collapse processes. In other words, in the standard treatments, the spectrum would be determined by the inflationary theory (number of fields, kinetic terms, and interacting potentials), and the nature of the initial state, while in the approach we have been advocating a novel source of statistical anomalies, is provided by the details of the modification of quantum theory by the dynamics of collapse.

One example of these novel possibilities is provided by the study of the details of the mode by mode collapse within the semiclassical treatment of the problem as developed in Ref. [13]. In that work, it was found the collapse of a mode with comoving wave vector $\vec{k}_{0}$ must be tied with the modification of the state of the field in the higher harmonics of that mode. It was found, in particular, that the effect would be stronger for mode $2 \vec{k}_{0}$. This, in turn, leads to the consideration of the possibility of strong correlations in the collapse parameters of the two modes, an effect that would produce a particular type of exotic correlations-it is unclear if they should be called non-Gaussianities as they involve modifications of the two-point functions-something that would produce a particular kind of signature in the CMB [14].

The organization of this manuscript is as follows: In Sec. II, we offer a preliminary discussion of the posture we advocate regarding the emergence of structure and its implications for the statistical analysis of the CMB and some aspects of the usual approach focusing on the aspects we consider to be conceptually unclear. In Sec. III, we review the standard picture for primordial nonGaussianities. In Sec. IV, we review the collapse models description for the inflationary origin of the seeds of the cosmic structure. In Sec. V, we focus on the statistical aspects as seen from our perspective of the primordial inhomogeneities, propose new characterizations of the non-Gaussianities, and discuss new measures to be associated with the bispectrum. Finally, in Sec. VI, we discuss our findings. We use conventions in which the signature of the space-time metric is $(-,+,+,+)$ and units where $c=1$ but will keep the gravitational constant $G$ and $\hbar$ appearing explicitly throughout the paper.

\section{SOME PRELIMINARIES ON THE EMERGENCE OF FEATURES OF THE CMB AND STATISTICAL CONSIDERATIONS}

Let us start this section by noting that, in the usual accounts, it is hard to pinpoint where exactly the statistical aspects enter at the theoretical level, how that is connected to the kind of statistics one considers at the observational level, and which kind of statistics one is dealing with. That is, in the standard approach, our specific Universe is not described in any sense (not even in terms of unknown yet explicitly identified quantities), and the randomness that one invokes, as characterizing the relation of theory and observation, lies hidden in unspecified aspects associated with the vagueness of the interpretations. In other words, one cannot identify the random variables; one does not know how many there are, and one cannot say how exactly the various elements of the ensemble of Universes differ from each other. One imagines an ensemble of universes and assumes that their collective departure from H\&I is somehow characterized by the H\&I vacuum state or the state that results form the unitary evolution thereof (despite such a state being homogeneous and isotropic). One then considers that the ensemble is being described, while each of the individual elements of the ensemble cannot be described, or that its description is irrelevant.

Within such setting, one proceeds to make, either explicitly or implicitly, the assumption that statistics over such an ensemble correspond to the statistics, over time, over space, or over orientation, in our particular Universe. In fact, one assumes that they are all equivalent. It should be clear that such assumptions are, therefore, taken to say something about the individual element of the ensemble, and it is not completely clear what it is. If our Universe is not described by the quantum state we use in our equations, what can we say about it? In order to look for justification and clarification of such identifications, we must turn to the quantum theory from which one expects to extract the predictions. The problem is that, while quantum theory has a clear and workable interpretation (even if not completely satisfactory [15]) for dealing with laboratory experiments (the Copenhagen interpretation), for which the measuring devices and observers can be taken as clearly identified, for the case of the cosmological problem at hand, we are faced with a situation deprived of such entities that normally provide an interpretation.

Thus, the issue we will be addressing cannot be turned into one of "measurement," while implicitly assuming that such concept can be used in the delicate quantum mechanical context examined in this paper. This is simply because as we have already noted, cosmology needs to account for the emergence of the conditions ${ }^{6}$ that make such things as observers and apparatuses possible to start with.

In order to fully and satisfactorily address the problem at hand, it seems we need to be able to point out "what exactly is wrong with the argument leading to the conclusion drawn above. In other words, where does nature

\footnotetext{
${ }^{6}$ Primordial inhomogeneities are supposed to evolve into galaxies and galaxy clusters, and within galaxies, stars and planets are supposed to arise by gravitational collapse, and then life is supposed to arise in the appropriate circumstances on some planets, particularly on Earth.
} 
deviate from the theory leading to the erroneous conclusion that our Universe is, even today, at the fundamental quantum level, perfectly homogeneous and isotropic?" It follows that such explanation must indicate where the ordinary $U$ evolution-with the symmetry preserving Hamiltonian-breaks down.

We can easily see that none of the proposals to deal with the issue, and which are based on the standard paradigms, can single out any point where that breakdown might occur or, much less, point to a physical reason for that departure from standard quantum theory (we turn the interested reader toward Appendix $\mathrm{C}$, where we explore in more detail these issues and justify more precisely our point of view).

This has led us to take a view that ties this problem with the ideas advocated by L. Diosi and R. Penrose, which argue $[16,17]$ that quantum theory should itself suffer modifications as a result of its combination with the fundamental theory of space-time structure. ${ }^{7}$ Among the aspects of the theory that would be substantially affected according to those views are those related to the reduction postulate (or $R$ process) and its contrast with the unitary evolution (or $U$ process) controlled by Schrödinger's equation. In fact, the issue of dynamical quantum reduction has received a lot of attention within the community working in foundational aspects of quantum theory, and there are, in the existing literature, several rather well-defined proposals in this regard, such as those in Refs. [15,16,18-20].

The proposal behind our work is based on the hypothesis that a dynamical collapse of the wave function lies behind the breakdown of the initial homogeneity and isotropy. In other words, a nonunitary "jump" in the quantum state of the system plays a role in transforming the inflaton vacuum into a quantum state that lacks the translational and rotational symmetries of the former state.

It goes without saying that we cannot, at this stage, try or hope to point out the precise physical origin of such dynamical collapse. ${ }^{8}$ However, once one has accepted that something of this sort is occurring, one can parametrize its basic characteristics and use the relevant observational data to infer something about the nature of the novel physics that must lie behind such phenomena. This has been the basic attitude behind the program started in

\footnotetext{
${ }^{7}$ This is what is often thought of as quantum gravity. We did not use that term because that often presupposes that one is considering the relevant theory to be simply the adaptation of general relativity to the standard quantum theory, while what one has in mind, when following Diosi and Penrose's ideas, is something much more distant from known physics, involving, as indicated, modifications of quantum theory itself.

${ }^{8}$ In particular, collapse theories are known to face, in principle, serious difficulties with Lorentz and general covariance and issues related to conservation laws. However, important advances have been made in addressing both classes of issues (Refs. [21,22]), even if we cannot say we have at our disposal anything resembling a completely satisfactory theory.
}

Ref. [4]. We should emphasize, that although most of our work has centered on that rather simplistic collapse model developed specifically for the cosmological problem at hand, the discussion of most of this paper would apply equally to more general models and, in particular, to approaches based on exciting proposals like GhirardiRimini-Weber [18] and continuous spontaneous localization [23]. In fact, some recent works are devoted to the adaptation of the continuous spontaneous localization theory for its application to the problem of the emergence of inhomogeneities and anisotropies in cosmology [24,25].

Here, we want to focus on the impact of such ideas on the statistical study of the CMB. We will discuss some delicate interpretational aspects related to quantum theory, its implicit usage in the standard approach to the study of the $\mathrm{CMB}$, and its characterization in terms of a spectra as well as the accounts of the origin of cosmic structure. We will briefly explore here, for the first time, some of the basic differences associated with statistical considerations, between those tied to the usual approach and those appropriate to our proposal.

In order to make things a bit more explicit, let us start by reminding the reader that in the standard approaches, the study of the statistical nature of the problem is based on the study of the statistical $n$-point functions of the Newtonian potential, $\overline{\Psi\left(x_{1}\right) \ldots \Psi\left(x_{n}\right)}$, with the overline denoting the average over an ensemble of universes. Having no access to such ensemble, one needs to face the issue of what the relationship between those $n$-point functions and the quantities we actually measure is. Moreover, one needs to consider how these quantities are connected with the quantum $n$-point functions. The usual approach [26,27] relies on the identification

$$
\overline{\Psi\left(x_{1}\right) \ldots \Psi\left(x_{n}\right)}=\left\langle 0\left|\hat{\Psi}\left(x_{1}\right) \ldots \hat{\Psi}\left(x_{n}\right)\right| 0\right\rangle,
$$

where $\left\langle 0\left|\hat{\Psi}\left(x_{1}\right) \ldots \hat{\Psi}\left(x_{n}\right)\right| 0\right\rangle$ is a standard quantum mechanical $n$-point function for the quantum field operators (corresponding to the vacuum state at hand). That is, one is making the identification of quantum and statistical $n$-point functions. As we said, the latter are naturally associated with an ensemble of universes, all of which, even if real, are unaccessible to us. The usual line of argument continues by invoking ergodic arguments, to make a further connection between ensemble averages and time averages, with other vague arguments indicating one might replace the latter with spatial averages and often turning, in practice, to orientation averages. On the other hand, at the quantum level, the interpretation is, as we noted before, even more problematic. In the standard laboratory situations, one has an apparatus designed to measure a certain observable $\mathcal{O}$, and quantum theory then indicates that, in each individual measurement, one would obtain an eigenvalue of the corresponding operator. Furthermore, immediately after the measurement, the individual system is taken to be in the state corresponding to the resulting 
eigenvalue (as immediate repetition of the same measurement in such an individual system gives, with probability 1 , the exact same value). The sudden change in the state of the system is known as the state function reduction or wave function collapse and is thought as being brought up by the measurement (in fact, the interpretation is not fully satisfactory, but we have become used to the fact that in laboratory situations it works). Moreover, the quantum expectation value of the observable $\langle\xi|\mathcal{O}| \xi\rangle$ in the state $|\xi\rangle$ (the system's state before the measurement) should be equal to the average of the observed values of the corresponding quantity, over a large enough ensemble of identical systems. It is important to note here that such an interpretational scheme works only as long as a clearly identified measurement is involved, as one essential aspect of the nature of the quantum world is that one cannot consistently adopt a point of view advocating that the measurement simply served to reveal a preexisting value of such a quantity (see, for instance, Ref. [28]).

Let us illustrate these and other related issues by considering the simplest place where one can appreciate the problematic aspects of such identifications: the case of the one-point function. Let us focus here on the standard treatment that relies on the so-called Mukhanov-Sasaki variables, defined by

$$
u \equiv \frac{a \Psi}{4 \pi G \dot{\phi}_{0}}, \quad v \equiv a\left(\delta \phi+\frac{\dot{\phi}_{0}}{\mathcal{H}} \Psi\right),
$$

where $\Psi$ is the metric perturbation known as the Newtonian potential, $\dot{\phi}_{0}$ is the derivative of the background inflaton with respect to conformal time $\eta, \delta \phi$ is the perturbation in the inflaton field, $a$ is the scale factor, and $\mathcal{H} \equiv \frac{\dot{a}}{a}$ (related to the standard Hubble parameter $H$ through $\mathcal{H}=a H)$. Einstein's equations then lead to $\Delta u=z\left(\frac{v}{z}\right)^{\circ}$ and $v=\frac{1}{z}(z u)^{\circ}$, where $z \equiv \frac{a \dot{\phi}_{0}}{\mathcal{H}}$. Given the equations of motion, the Newtonian potential can thus be expressed in terms of the field $v(\vec{x}, \eta)$ and its momentum conjugate $\pi_{v}(\vec{x}, \eta)=\dot{v}(\vec{x}, \eta)$. The expression for the corresponding Fourier components is

$$
\Psi_{\vec{k}}(\eta)=-\frac{\sqrt{4 \pi G \epsilon} H}{k^{2}}\left(\pi_{v \vec{k}}(\eta)-\frac{\dot{z}}{z} v_{\vec{k}}(\eta)\right)
$$

where $\epsilon$ is the so-called slow-roll parameter $\epsilon \equiv$ $1-\dot{\mathcal{H}} / \mathcal{H}^{2}$.

We are interested in the temperature anisotropies of the CMB observed today on the celestial two-sphere, which are related to the inhomogeneities in the Newtonian potential on the last scattering surface,

$$
\frac{\delta T}{T_{0}}(\theta, \varphi)=\frac{1}{3} \Psi\left(\eta_{D}, \vec{x}_{D}\right) .
$$

The data are described in terms of the coefficients $\alpha_{l m}$ of the multipolar series expansion

$$
\begin{aligned}
\frac{\delta T}{T_{0}}(\theta, \varphi) & =\sum_{l m} \alpha_{l m} Y_{l m}(\theta, \varphi), \\
\alpha_{l m} & =\int \frac{\delta T}{T_{0}}(\theta, \varphi) Y_{l m}^{*}(\theta, \varphi) d \Omega ;
\end{aligned}
$$

here, $\theta$ and $\varphi$ are the coordinates on the celestial two-sphere, with $Y_{l m}(\theta, \varphi)$ as the spherical harmonics.

The value for the quantities $\alpha_{l m}$ are then given by

$$
\alpha_{l m}=\frac{4 \pi i^{l}}{3} \int \frac{d^{3} k}{(2 \pi)^{3}} j_{l}\left(k R_{D}\right) Y_{l m}^{*}(\hat{k}) \Delta(k) \Psi_{\vec{k}}\left(\eta_{R}\right)
$$

with $j_{l}\left(k R_{D}\right)$ as the spherical Bessel function of order $l ; \eta_{R}$ is the conformal time of reheating, which can be associated with the end of the inflationary regime, and $R_{D}$ is the comoving radius of the last scattering surface. We have explicitly included the modifications associated with latetime physics encoded in the transfer functions $\Delta(k)$.

Now, the problem is that, if we compute the expectation value of the right-hand side (i.e., identifying $\langle\hat{\Psi}\rangle=\Psi$ ) in the vacuum state $|0\rangle$, we obtain 0 , while it is clear that for any given $l, m$, the measured value of this quantity is not $0 .{ }^{9}$ That is, if we rely in this case on the one-point function and the standard identification, we find a large conflict between expectation and observation. We might even be tempted to say that evidence of non-Gaussianity has already been observed in each measurement of a particular $\alpha_{l m}$. This is, of course, not what one wants. Advocates of the standard approach would indicate that $\left\langle\alpha_{l m}\right\rangle=0$ is not to be taken as "the prediction of the approach" regarding our Universe and that this would only hold for an ensemble of universes. The issue, of course, is what precise interpretational posture regarding the theory can be used to justify this, while at the same time justifying the positions taken vis-à-vis the other quantities that emerge from the theory (such as the higher $n$-point functions). A theory that depends on a case by case adaptation of an interpretational rule is not a very satisfactory theory. However, this makes clear that disentangling the various statistical aspects (ensemble statistics; space and time statistics, including orientation statistics; and, finally, the nature of the assumed connection of quantum and statistical aspects) and making explicit the assumptions underlying the identifications, as well as the expected limitations, is paramount to avoid confusion and to allow the judging of a theory on its true merits.

As a matter of fact, it seems clear that anything that can be considered as a satisfactory approach should enable one to understand what exactly is wrong with the above

\footnotetext{
${ }^{9} \mathrm{We}$ are ignoring the remote possibility that, just by coincidence, and for some specific $l$ and $m$, the quantity $\alpha_{l m}$ would vanish within the observational margin of error. As can be seen in Sec. IV, according to our point of view, that would require a remarkable cancelation between terms determined by a large collection of random numbers.
} 
argument. First, let us note that, just as the Fourier transform of a function is a weighted average (with weight $\left.e^{i \vec{k} \cdot \vec{x}}\right)$, so are the spherical harmonic transforms of functions. Thus, $\alpha_{l m}$ is a weighted average over the last scattering surface (cosmologists often refer to the average over the sky) because it is an integral over the celestial two-sphere of $\frac{\delta T}{T}$ weighted with a given function, the $Y_{l m}$. The common argument in the literature, as we have noted, indicates that averaging over the sky justifies the identification of observations with quantum expectation values.

In other words, the argument indicates that the relevant prediction (obtained in terms of quantum expectation values) concerns the ensemble averages, and these should be equal to spatial averages and the latter to averages over the sky. However, apparently, this should not hold for weighted averages over the sky (otherwise, all the $\alpha_{l m}$ 's would be 0 ). If not, why not? There seems to be no clear answer.

Namely, if we take the theoretical estimate as

$\alpha_{l m}^{\mathrm{th}}=\frac{4 \pi i^{l}}{3} \int \frac{d^{3} k}{(2 \pi)^{3}} j_{l}\left(k R_{D}\right) Y_{l m}^{*}(\hat{k}) \Delta(k)\left\langle 0\left|\hat{\Psi}_{\vec{k}}\left(\eta_{R}\right)\right| 0\right\rangle=0$

and compare it with the measured quantity $\alpha_{l m}^{\text {obs }}$, we would find a large discrepancy. The answer, within the standard accounts, would need to be that, for some reason, in order to be allowed to make identifications, we should invoke a further averaging: the average over orientations. Only then would we have any confidence that our estimates are reliable. Now, let us ask ourselves the question of why this should be; it seems completely unclear. Anyhow, the point is that we would be asked to compute

$$
\overline{\alpha_{l}}=\frac{1}{2 l+1} \sum_{m} \alpha_{l m},
$$

and we would then expect this quantity to be zero.

We need to confront the following issues:

(i) Why is that so? Why can this average be expected to yield zero but not each individual $\alpha_{l m}$ as in Eq. (7)?

(ii) Empirically, does this hold? In other words, is the actual average of observed complex quantities in Eq. (8), in fact, zero, or is it not?

Regarding the first question, it seems imperative to choose a suitable interpretational framework in order to be able to decide a priori what the appropriate identifications are and also to be able to evaluate whether or not we have a good theoretical understanding. It appears that, in the standard way of looking at the issue, there is really no justification to expect anything but the vanishing of each $\alpha_{l m}$. We must avoid getting confused with the notion that quantum theory involves uncertain predictions. The point is that the only part of quantum theory that involves such indeterminism is the measurement process, and we do not want to call upon that in this particular situation. It is true that, even in ordinary laboratory situations, the "measurement problem" is quite unsettling. However, in the case at hand, the problem is exacerbated because we cannot even contemplate any physical observer or measuring device existing prior to the emergence of the seeds of structure. Thus, we cannot even rely on our old battle tool: the Copenhagen interpretation, which explains the nonvanishing of those quantities that predates both the growth of galaxies and the existence of "observers and measuring devices."

Regarding the second issue, we would like to comment the work of Armendariz-Picon [29], which starts to address (albeit in a rather limited way, because the analysis is done for a very small number of values of $l$ ) that question. The results of this work indicate that the $\overline{\alpha_{l}}$ are small (1 order of magnitude smaller than the variance of the $\alpha_{l m}$, that is, $\sqrt{C_{l}}$ ), and that seems reassuring. But is this sufficient? Is that what we should expect according to our theory? Why? Should it not be zero up to the actual experimental errors ${ }^{10}$ in the observations? Evidently, these are just rhetorical questions, raised only to show that it is easy to be confused regarding the comparisons of theory and observations, if one accepts, without questioning, the usual arguments given by the standard approach. It seems evident that, in order to have a clear answer to those questions, one needs to have a precise and unambiguous characterization of what exactly the mapping between the theory and the measured quantities is. Actually, it seems one would need to consider those comparisons as tests of whether the identifications one is making are or are not appropriate ones.

It is our view, as advocated in Refs. [4,5], that the standard paradigm has no satisfactory answers to these issues. We hope this brief discussion serves to illustrate the problem we must face concerning the identification of theoretical predictions and observations in the situation at hand.

We end this section by reminding the reader that, if one wants to consider the average value of any quantity, it is imperative to specify over which set the average is defined. There are just no "averages" as absolute concepts. In the remainder of the manuscript, we will make an important differentiation between averages over ensembles of universes, averages over a spacelike hypersurface, averages over the last scattering surface, and averages over orientations. The question we want to address is how we are able

\footnotetext{
${ }^{10}$ Here, we should be careful in considering the sources of error: As in any measurement, we have the systematic errors and the statistical errors associated with uncontrolled disturbances, but we should not confuse statistics over several determinations of a specific $\alpha_{l m}$, say, with different experimental runs or with different satellites, and the statistics for a fixed $l$ over the orientation number $m$. For a fixed value of $l$, the variability of $\alpha_{l m}$ with $m$ should not, in our view, be taken as some statistical error but as truly valuable data containing valuable information about the physics behind the emergence of the seeds of cosmic structure.
} 
to compare the theoretical estimates, based on quantum expectation values for some quantities, with measured values of related quantities. The approach we will be primarily focusing on is the one pioneered in Ref. [4] and which seems to have more potential for dealing univocally with such questions than the standard one.

\section{THE STANDARD PICTURE FOR THE PRIMORDIAL NON-GAUSSIANITIES}

This section will briefly review the standard accounts on the primordial non-Gaussianities following closely Refs. [30-32]. There is absolutely no original work in this section or any extensive discussion of our views (just a few relevant comments); we simply present here the usual treatment on the subject following what is commonly found in the literature, in order to compare it with our own approach, and discuss the main differences. For more details and derivations, we refer the reader to the comprehensive review by Komatsu [33], Bartolo et al. [34], and the references cited therein.

Historically, non-Gaussianity, as a test of the accuracy of perturbation theory, was first suggested by Allen et al. [35]. However, most of its importance to date relies on the premise that it will play a leading role in furthering our understanding of two fundamental aspects of cosmology and astrophysics [36]:

(i) the physics of the very early Universe that created the primordial seeds for large-scale structures,

(ii) the subsequent growth of structures via gravitational instability and gas physics at later times.

Within the standard approach, by non-Gaussianity, one refers to any small deviations in the observed fluctuations from the random field of linear, Gaussian, curvature perturbations. The curvature perturbations, $\Psi$, generate the CMB anisotropy, $\delta T / T$. The linear perturbation theory gives a linear relation between $\Psi$ and $\delta T / T$ on large scales (where the Sachs-Wolfe effect dominates) at the decoupling epoch, i.e., $\delta T / T \sim(1 / 3) \Psi$. It follows from the relation, $\delta T \propto \Psi$, that if $\Psi$ is Gaussian, then $\delta T$ is Gaussian, but what exactly does one mean by Gaussian at the observational level?

One of the most important results of the inflationary paradigm is that the $\mathrm{CMB}$ anisotropy arises due to curvature perturbations, which, in turn, are produced by quantum fluctuations. In the standard single-field slow-roll scenario, these fluctuations are due to fluctuations of the inflaton field itself, when it slowly rolls down its potential $V(\phi)$. Within this approach, the primordial perturbation is Gaussian; in other words, its Fourier components are uncorrelated and have random phases. When inflation ends, the inflaton $\phi$ oscillates about the minimum of its potential and decays, thereby reheating the Universe.

In the inflationary paradigm, the perturbations of the field $\delta \phi$ and the perturbations of the curvature $\Psi$ are treated as standard quantum fields, ${ }^{11}$ evolving in a classical quasi-de Sitter background space-time. The quantity of observational interest is called the power spectrum of the curvature perturbation $P_{\Psi}(k, \eta)$. The power spectrum is obtained from

$$
\langle 0|\hat{\Psi}(\vec{x}, \eta) \hat{\Psi}(\vec{y}, \eta)| 0\rangle,
$$

where $|0\rangle$ is called the Bunch-Davies vacuum and represents the initial state of the field $\hat{v}$, which is the MukhanovSasaki field variable defined in Eq. (2) (for a discussion about the symmetric properties of the Bunch-Davies vacuum, see Appendix A).

It is precisely at this step where a subtle issue arises, namely, that, in the standard picture, one is given various and distinct arguments (e.g., decoherence, horizon crossing, many worlds interpretation of quantum mechanics, etc.) to accept the identification

$$
\langle 0|\hat{\Psi}(\vec{x}, \eta) \hat{\Psi}(\vec{y}, \eta)| 0\rangle=\overline{\Psi(\vec{x}, \eta) \Psi(\vec{y}, \eta)},
$$

where $\Psi(\vec{x}, \eta)$ now stands as a classical stochastic field and the overline denotes the average over an ensemble of universes. In other words, the value of the field $\Psi$ in each point $(\vec{x}, \eta)$ varies from each one of the members of the ensemble of "universes," with a variance $\overline{\Psi^{2}}$. Therefore, the power spectrum $P_{\Psi}(k, \eta)$ is defined in terms of the Fourier components of $\Psi(\vec{x}, \eta)$ by

$$
\overline{\Psi_{\vec{k}}(\eta) \Psi_{\vec{k}^{\prime}}(\eta)} \equiv(2 \pi)^{3} \delta\left(\vec{k}+\vec{k}^{\prime}\right) P_{\Psi}(k, \eta) .
$$

Consequently, the power spectrum is related to the twopoint function through

$$
\overline{\Psi(\vec{x}, \eta) \Psi(\vec{y}, \eta)}=\int_{0}^{\infty} \frac{d k}{k} \mathcal{P}_{\Psi}(k, \eta) \frac{\sin k r}{k r},
$$

with $r \equiv|\vec{x}-\vec{y}|$, and we also used the definition of the dimensionless power spectrum $\mathcal{P}_{\Psi}(k, \eta) \equiv$ $P_{\Psi}(k, \eta) k^{3} / 2 \pi^{2}$. The variance $\overline{\Psi^{2}}$ is given by

$$
\overline{\Psi^{2}(\vec{x}, \eta)}=\int_{0}^{\infty} \frac{d k}{k} \mathcal{P}_{\Psi}(k, \eta) .
$$

The expression (13) diverges generically. In particular, we know that the spectrum of the primordial curvature perturbation is roughly $P_{\Psi}(k, \eta) \propto k^{-3}$. That is, $\mathcal{P}_{\Psi}(k, \eta)$ is nearly constant (i.e., independent of $k$ ); therefore, Eq. (13) diverges in a logarithmic way for $k \rightarrow 0$ and $k \rightarrow \infty$. The way the standard pictures deal with this issue [27] is to establish a $k_{\max }$ equal to the "horizon" and work in a cubic box of physical size $a L$ much larger than the Hubble radius. Thus,

$$
\overline{\Psi^{2}(\vec{x}, \eta)} \simeq \mathcal{P}_{\Psi}(\eta) \int_{L^{-1}}^{a H} \frac{d k}{k}=\mathcal{P}_{\Psi}(\eta) \ln \frac{a L}{H^{-1}} .
$$

That is, in order to avoid the divergence in $\overline{\Psi^{2}}$, one is forced to introduce some particular values of $k$ as cutoffs

\footnotetext{
${ }^{11}$ In fact, they are both part of a unified field $v$.
} 
(for a detailed discussion related to this fact, see Appendix B).

The question that arises now is how can we evaluate any average over an ensemble of universes if we have observational access to just one-our own-Universe. The obvious answer is that we cannot. Normally, one is presented with ergodic arguments indicating that averages over time should be equated with ensemble averages. However, ergodicity relies on equilibrium, and the inflationary regime is not one of equilibrium. Furthermore, ignoring that issue, we would need to find an argument justifying the identification of time averages and spatial averages, presumably to be carried over the hypersurface corresponding to the time of decoupling. Then, we need to make sure our argument applies only to direct averages and not to weighted averages, as we discussed in the introduction. And finally, as we do not have access (at least using the CMB) to that whole hypersurface, nor to any large open region within it, but only to the portion of it that intersects our past light cone (the two-sphere known as the last scattering surface), we must find some argument indicating we can replace such spatial averages with averages over orientations.

In the next subsection, we will show how our approach deals with these questions. In the remainder of this section, we will accept the validity of Eq. (10) and ignore those issues.

If $\Psi(\vec{x}, \eta)$ is Gaussian, ${ }^{12}$ then the two-point correlation function (9) specifies all the statistical properties of $\Psi(\vec{x}, \eta)$, for the two-point correlation function is the only parameter in a Gaussian distribution. If it is not Gaussian, then we need higher-order correlation functions to determine the statistical properties.

For instance, a nonvanishing three-point function ${ }^{13}$

$$
\overline{\Psi(\vec{x}, \eta) \Psi(\vec{y}, \eta) \Psi(\vec{z}, \eta)}
$$

is an indicator of non-Gaussian features in the cosmological perturbations. The Fourier transform of the three-point function is called the bispectrum ${ }^{14}$ and is defined as

$$
\overline{\Psi_{\vec{k}_{1}} \Psi_{\vec{k}_{2}} \Psi_{\vec{k}_{3}}} \equiv(2 \pi)^{3} \delta\left(\vec{k}_{1}+\vec{k}_{2}+\vec{k}_{3}\right) B_{\Psi}\left(k_{1}, k_{2}, k_{3}\right) .
$$

The importance of the bispectrum comes from the fact that it represents the lowest-order statistics able to distinguish non-Gaussian from Gaussian perturbations.

\footnotetext{
${ }^{12}$ That is, there exists some physical mechanism for which the quantum variable $\hat{\Psi}(\vec{x}, \eta)$ becomes a classical stochastic field $\Psi(\vec{x}, \eta)$ with Gaussian distribution.

${ }^{13}$ Just as in the case of the two-point correlation function, the standard approach relies on the identification

$\langle 0|\hat{\Psi}(\vec{x}, \eta) \hat{\Psi}(\vec{y}, \eta) \hat{\Psi}(\vec{z}, \eta)| 0\rangle=\overline{\Psi(\vec{x}, \eta) \Psi(\vec{y}, \eta) \Psi(\vec{z}, \eta)}$.

${ }^{14}$ In the following, we will not write the explicit dependance of the conformal time $\eta$ unless it leads to possible confusion.
}

The delta function in Eq. (17) enforces the triangle condition, that is, the constraint that the wave vectors in Fourier space must close to form a closed triangle, i.e., $\vec{k}_{1}+\vec{k}_{2}+\vec{k}_{3}=0$. Different inflationary models predict a maximal signal for different triangle configurations. The standard approach of the study of the structure of the bispectrum is usually done by plotting the magnitude of $B_{\Psi}\left(\vec{k}_{1}, \vec{k}_{2}, \vec{k}_{3}\right)\left(k_{2} / k_{1}\right)^{2}\left(k_{3} / k_{1}\right)^{2}$ (with $\left|\vec{k}_{i}\right| \equiv k_{i}$ ) as a function of $k_{2} / k_{1}$ and $k_{3} / k_{1}$ for a given $k_{1}$, with a condition that $k_{1} \geq k_{2} \geq k_{3}$ is satisfied. The usual classification of various shapes of the triangles uses the following names: squeezed $\quad\left(k_{1} \simeq k_{2} \gg k_{3}\right)$, elongated $\left(k_{1}=k_{2}+k_{3}\right)$, folded $\left(k_{1}=2 k_{2}=2 k_{3}\right)$, isosceles $\left(k_{2}=k_{3}\right)$, and equilateral $\left(k_{1}=k_{2}=k_{3}\right)$. Within the cosmology community [37-39], these shapes of non-Gaussianity are potentially a powerful probe of the mechanism that creates the primordial perturbations.

One of the first (and most popular) ways to parametrize non-Gaussianity phenomenologically was via a small nonlinear correction to the linear Gaussian perturbation [40,41],

$$
\begin{aligned}
\Psi(\vec{x}, \eta) & =\Psi_{\mathrm{L}}(\vec{x}, \eta)+\Psi_{\mathrm{NL}}(\vec{x}, \eta) \\
& \equiv \Psi_{\mathrm{L}}(\vec{x}, \eta)+f_{\mathrm{NL}}^{\mathrm{loc}}\left[\Psi_{\mathrm{L}}^{2}(\vec{x}, \eta)-\overline{\Psi_{\mathrm{L}}^{2}(\vec{x}, \eta)}\right]
\end{aligned}
$$

where $\Psi_{\mathrm{L}}(\vec{x}, \eta)$ denotes a linear Gaussian part of the perturbation, and the variance $\overline{\Psi_{\mathrm{L}}^{2}(\vec{x}, \eta)}$ is implemented in the same sense as presented in Eq. (14). Henceforth, $f_{\mathrm{NL}}^{\text {loc }}$ is called the local nonlinear coupling parameter and determines the "strength" of the primordial nonGaussianity. This parametrization of non-Gaussianity is local in real space and, therefore, is called local nonGaussianity. In this local model, the contributions from "squeezed" triangles are dominant, that is, with, e.g., $k_{3} \ll k_{1}, k_{2}$. Using Eqs. (18) and (17), the bispectrum of local non-Gaussianity may be derived:

$$
\begin{aligned}
B_{\Psi}\left(\vec{k}_{1}, \vec{k}_{2}, \vec{k}_{3}\right)= & 2 f_{\mathrm{NL}}^{\mathrm{loc}}\left[P_{\Psi}\left(\vec{k}_{1}\right) P_{\Psi}\left(\vec{k}_{2}\right)+P_{\Psi}\left(\vec{k}_{2}\right) P_{\Psi}\left(\vec{k}_{3}\right)\right. \\
& \left.+P_{\Psi}\left(\vec{k}_{3}\right) P_{\Psi}\left(\vec{k}_{1}\right)\right] .
\end{aligned}
$$

In the standard picture, the non-Gaussianity produced by many single-field slow-roll models is considered small and likely unobservable. However, a relatively large, possibly detectable, amount of non-Gaussianity can be expected when any of the following conditions are violated [34,36,42,43]:

(i) Single Field-There was only one quantum field responsible for driving inflation.

(ii) Canonical Kinetic Energy-The kinetic energy of the quantum field is such that the speed of propagation of fluctuations is equal to the speed of light.

(iii) Slow Roll-The evolution of the field was always very slow compared to the Hubble time during inflation. 
(iv) Initial Vacuum State - The quantum field was in the preferred "Bunch-Davies vacuum" state.

\section{A. Non-Gaussianity in the CMB}

In this subsection, we present the standard connection between the primordial bispectrum at the end of inflation and the observed bispectrum of CMB anisotropies.

\section{Theoretical predictions for the CMB bispectrum from inflation}

As we mentioned in Sec. I, the temperature anisotropies are represented using the $\alpha_{l m}$ coefficients of a spherical harmonic decomposition of the celestial sphere,

$$
\frac{\delta T}{T_{0}}(\theta, \varphi)=\sum_{l m} \alpha_{l m} Y_{l m}(\theta, \varphi),
$$

and the curvature perturbation $\Psi$ is imprinted on the CMB multipoles $\alpha_{l m}$ by a convolution involving the called transfer functions $\Delta(k)$ representing the linear perturbation evolution, through Eq. (6):

$$
\alpha_{l m}=\frac{4 \pi i^{l}}{3} \int \frac{d^{3} k}{(2 \pi)^{3}} j_{l}\left(k R_{D}\right) Y_{l m}^{*}(\hat{k}) \Delta(k) \Psi_{\vec{k}}\left(\eta_{R}\right) .
$$

The CMB bispectrum, also called the angular bispectrum, is defined as the three-point correlator of the $\alpha_{l m}$ :

$$
B_{m_{1} m_{2} m_{3}}^{l_{1} l_{2} l_{3}} \equiv \overline{\alpha_{l_{1} m_{1}} \alpha_{l_{2} m_{2}} \alpha_{l_{3} m_{3}}} .
$$

At this point, the standard picture leads us to another subtle issue; that is, the overline in Eq. (21) denotes, in principle, an average over an ensemble of universes. In reality, we cannot measure the ensemble average of the angular harmonic spectrum, as we have access to just one realization, say, the collection of complex numbers: $\left\{\alpha_{l_{1} m_{1}}, \alpha_{l_{2} m_{2}}, \ldots, \alpha_{l_{n} m_{n}}\right\}$. In order to overcome this issue, the standard approach relies on the ergodic assumption [27]. The ergodicity of a system refers to that property of a process by which the average value of a system's characteristic, measured over time, is the same as the average value measured over an appropriately constructed ensemble. If one accepts the common supposition that the inflationary perturbation is indeed ergodic, then one expects the volume average of the fluctuations to behave like the ensemble average: The universe may contain regions where the fluctuation is atypical, but with high probability, most regions contain fluctuations with a root-mean-square amplitude close to $\sigma$ [44]. Therefore, the probability distribution on the ensemble, which is encoded in Eq. (21), translates to a probability distribution on smoothed regions of a determined size within our own Universe.

After the above analysis, we continue with the calculation relating the primordial bispectrum with the angular bispectrum. By substituting Eq. (6) in Eq. (21), one obtains

$$
\begin{aligned}
& B_{m_{1} m_{2} m_{3}}^{l_{1} l_{2} l_{3}}=\left(\frac{4 \pi}{3}\right)^{3} i^{l_{1}+l_{2}+l_{3}} \int \frac{d^{3} k_{1}}{(2 \pi)^{3}} \frac{d^{3} k_{2}}{(2 \pi)^{3}} \frac{d^{3} k_{3}}{(2 \pi)^{3}} \Delta\left(k_{1}\right) \Delta\left(k_{2}\right) \Delta\left(k_{3}\right) \times j_{l_{1}}\left(k_{1} R_{D}\right) j_{l_{2}}\left(k_{2} R_{D}\right) j_{l_{3}}\left(k_{3} R_{D}\right) \overline{\Psi_{\vec{k}_{1}}} \Psi_{\vec{k}_{2}} \Psi_{\vec{k}_{3}} Y_{l_{1} m_{1}}\left(\hat{k_{1}}\right) \\
& \times Y_{l_{2} m_{2}}\left(\hat{k_{2}}\right) Y_{l_{3} m_{3}}\left(\hat{k_{3}}\right) \\
& =\left(\frac{2}{3 \pi}\right)^{3} \int d k_{1} d k_{2} d k_{3}\left(k_{1} k_{2} k_{3}\right)^{2} B_{\Psi}\left(k_{1}, k_{2}, k_{3}\right) \Delta\left(k_{1}\right) \Delta\left(k_{2}\right) \Delta\left(k_{3}\right) \times j_{l_{1}}\left(k_{1} R_{D}\right) j_{l_{2}}\left(k_{2} R_{D}\right) j_{l_{3}}\left(k_{3} R_{D}\right) \\
& \times \int_{0}^{\infty} d x x^{2} j_{l_{1}}\left(k_{1} x\right) j_{l_{2}}\left(k_{2} x\right) j_{l_{3}}\left(k_{3} x\right) \times \int d \Omega_{\hat{x}} Y_{l_{1} m_{1}}(\hat{x}) Y_{l_{2} m_{2}}(\hat{x}) Y_{l_{3} m_{3}}(\hat{x}),
\end{aligned}
$$

where in the last line, we have integrated over the angular parts of the three $k_{i}$ and used the exponential integral form for the delta function that appears in the bispectrum definition (17). The last integral over the angular part of $\vec{x}$ is known as the Gaunt integral, which can be expressed in terms of Wigner 3-j symbols as

$$
G_{l_{1} l_{2} l_{3}}^{m_{1} m_{2} m_{3}} \equiv \int d \Omega_{\hat{x}} Y_{l_{1} m_{1}}(\hat{x}) Y_{l_{2} m_{2}}(\hat{x}) Y_{l_{3} m_{3}}(\hat{x})=\sqrt{\frac{\left(2 l_{1}+1\right)\left(2 l_{2}+1\right)\left(2 l_{3}+1\right)}{4 \pi}}\left(\begin{array}{ccc}
l_{1} & l_{2} & l_{3} \\
0 & 0 & 0
\end{array}\right)\left(\begin{array}{ccc}
l_{1} & l_{2} & l_{3} \\
m_{1} & m_{2} & m_{3}
\end{array}\right) \text {. }
$$

The fact that the bispectrum $B_{m_{1} m_{2} m_{3}}^{l_{1} l_{2} l_{3}}$ consists of the Gaunt integral, $G_{l_{1} l_{2} l_{3}}^{m_{1} m_{3} m_{3}}$, implies that the bispectrum satisfies the triangle conditions and parity invariance: $m_{1}+m_{2}+$ $m_{3}=0, l_{1}+l_{2}+l_{3}=$ even, and $\left|l_{i}-l_{j}\right| \leq l_{k} \leq l_{i}+l_{j}$ for all permutations of indices.

One, thus, can write

$$
B_{m_{1} m_{2} m_{3}}^{l_{1} l_{2} l_{3}}=G_{l_{1} l_{2} l_{3}}^{m_{1} m_{2} m_{3}} b_{l_{1} l_{2} l_{3}},
$$

where $b_{l_{1} l_{2} l_{3}}$ is an arbitrary real symmetric function of $l_{1}$, $l_{2}$, and $l_{3}$. This form, Eq. (24), is necessary and sufficient to construct generic $B_{m_{1} m_{2} m_{3}}^{l_{1} l_{2} l_{3}}$ satisfying rotational invariance; thus, in the literature, one encounters $b_{l_{1} l_{2} l_{3}}$ more frequently than $B_{m_{1} m_{2} m_{3}}^{l_{1} l_{2} l_{3}}$. The quantity $b_{l_{1} l_{2} l_{3}}$ is called the reduced bispectrum, as it contains all the physical information in $B_{m_{1}}^{l_{1} l_{2} l_{3} m_{3}}$. Since the reduced bispectrum does not contain the Wigner 3-j symbol, which merely ensures the triangle conditions and parity invariance, it is easier to calculate the physical properties of the theoretical bispectrum.

In the standard picture, one assumes that, if there is a nontrivial bispectrum, then it has arisen through a physical 
process that is statistically isotropic, ${ }^{15}$ so we can employ the angle-averaged bispectrum $B_{l_{1} l_{2} l_{3}}$ without loss of information, that is $[33,34]$,

$$
B_{l_{1} l_{2} l_{3}}=\sum_{m_{i}}\left(\begin{array}{ccc}
l_{1} & l_{2} & l_{3} \\
m_{1} & m_{2} & m_{3}
\end{array}\right) \overline{\alpha_{l_{1} m_{1}} \alpha_{l_{2} m_{2}} \alpha_{l_{3} m_{3}}} .
$$

We now can obtain a relation between the averaged bispectrum, $B_{l_{1} l_{2} l_{3}}$, and the reduced bispectrum, $b_{l_{1} l_{1} l_{2}}$, by substituting Eq. (24) into Eq. (25),

$B_{l_{1} l_{2} l_{3}}=\sqrt{\frac{\left(2 l_{1}+1\right)\left(2 l_{2}+1\right)\left(2 l_{3}+1\right)}{4 \pi}}\left(\begin{array}{ccc}l_{1} & l_{2} & l_{3} \\ 0 & 0 & 0\end{array}\right) b_{l_{1} l_{2} l_{3}}$,

where the identity

$$
\begin{aligned}
\sum_{\text {all } m} & \left(\begin{array}{ccc}
l_{1} & l_{2} & l_{3} \\
m_{1} & m_{2} & m_{3}
\end{array}\right) G_{l_{1} l_{2} l_{3}}^{m_{1} m_{2} m_{3}} \\
& =\sqrt{\frac{\left(2 l_{1}+1\right)\left(2 l_{2}+1\right)\left(2 l_{3}+1\right)}{4 \pi}}\left(\begin{array}{ccc}
l_{1} & l_{2} & l_{3} \\
0 & 0 & 0
\end{array}\right)
\end{aligned}
$$

was used. The reduced bispectrum obtained from Eq. (22) then takes the much simpler form

$$
\begin{aligned}
b_{l_{1} l_{2} l_{3}}= & \left(\frac{2}{3 \pi}\right)^{3} \int d k_{1} d k_{2} d k_{3}\left(k_{1} k_{2} k_{3}\right)^{2} B_{\Psi}\left(k_{1}, k_{2}, k_{3}\right) \Delta\left(k_{1}\right) \\
& \times \Delta\left(k_{2}\right) \Delta\left(k_{3}\right) \times j_{l_{1}}\left(k_{1} R_{D}\right) j_{l_{2}}\left(k_{2} R_{D}\right) j_{l_{3}}\left(k_{3} R_{D}\right) \\
& \times \int_{0}^{\infty} d x x^{2} j_{l_{1}}\left(k_{1} x\right) j_{l_{2}}\left(k_{2} x\right) j_{l_{3}}\left(k_{3} x\right) .
\end{aligned}
$$

This is the main equation for this section, since it explicitly relates the primordial bispectrum, predicted by the standard inflationary theories, to the averaged bispectrum [through Eq. (26)] obtained from the CMB angular bispec-

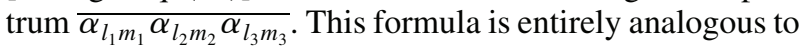
the well-known relation linking the primordial power spectrum $P_{\Psi}(k)$ and the CMB angular power spectrum $C_{l}$, i.e.,

$$
C_{l}=\frac{2}{9 \pi} \int k^{2} P_{\Psi}(k) \Delta^{2}(k) j_{l}^{2}\left(k R_{D}\right) d k
$$

\section{Measuring primordial non-Gaussianity from the CMB}

As we mentioned before, in most inflationary models, the parameter characterizing primordial non-Gaussianity is $f_{\mathrm{NL}}$. Thus, the next task within the standard picture is to estimate $f_{\mathrm{NL}}$ from the CMB data set. That is, one chooses the primordial model that one wants to test, characterizing it through its bispectrum shape. One then proceeds to estimate the corresponding amplitude $f_{\mathrm{NL}}^{\text {model }}$ from the data. If the final estimate is consistent with $f_{\mathrm{NL}}^{\text {model }}=0$,

\footnotetext{
${ }^{15}$ Although, it would be interesting, and possibly a more realistic approach to the problem, to proceed in the analysis without this assumption.
}

one concludes that no significant detection of the given shape is produced by the data, but one still determines important constraints on the allowed range of $f_{\mathrm{NL}}^{\text {model }}$. Note that, ideally, one would like to do more than just constrain the overall amplitude and reconstruct the entire shape from the data by measuring configurations of the bispectrum. However, the expected primordial signal is too small to allow the signal from a single bispectrum triangle to emerge over the noise. For this reason, one studies the cumulative signal from all the configurations that are sensitive to $f_{\mathrm{NL}}^{\text {model }}$.

Given the above analysis, the standard picture then makes use of estimation theory to extract an estimate for $f_{\mathrm{NL}}$ from the CMB data set. An unbiased bispectrum-based minimum variance estimator for the nonlinearity parameter can be written as $[45,46]$

$$
\begin{aligned}
\hat{f}_{\mathrm{NL}}= & \frac{1}{N} \sum_{l_{i} m_{i}}\left(\begin{array}{ccc}
l_{1} & l_{2} & l_{3} \\
m_{1} & m_{2} & m_{3}
\end{array}\right) \frac{B_{l_{1} l_{2} l_{3}}^{\mathrm{th}}}{\left(C_{l_{1}} C_{l_{2}} C_{l_{3}}\right)_{\mathrm{obs}}} \\
& \times\left(\alpha_{l_{1} m_{1}} \alpha_{l_{2} m_{2}} \alpha_{l_{3} m_{3}}\right)_{\mathrm{obs}},
\end{aligned}
$$

where $B_{l_{1} l_{2} l_{3}}^{\text {th }}$ is the angle-averaged theoretical CMB bispectrum for the model in consideration, with $f_{\mathrm{NL}}^{\text {th }}=1 ; C_{l}$ is the observed angular spectrum; and $\alpha_{l m}$ are the multipoles of the observed CMB temperature fluctuations. The normalization $N$ is calculated requiring the estimator to be "unbiased," i.e., the averaged value is equal to the "true" value of the parameter, $\left\langle\hat{f}_{\mathrm{NL}}\right\rangle=f_{\mathrm{NL}}$. If the bispectrum $B_{l_{1} l_{2} l_{3}}$ is calculated for $f_{\mathrm{NL}}=1$, then the normalization takes the following form:

$$
N=\sum_{l_{i}} \frac{\left(B_{l_{1} l_{2} l_{3}}\right)^{2}}{C_{l_{1}} C_{l_{2}} C_{l_{3}}}
$$

The estimator for non-Gaussianity (30) is then simplified using Eqs. (28) and (26) to yield

$$
\begin{aligned}
\hat{f}_{\mathrm{NL}}= & \frac{1}{N} \sum_{l_{i} m_{i}} \int d \Omega_{\hat{x}} Y_{l_{1} m_{1}}(\hat{x}) Y_{l_{2} m_{2}}(\hat{x}) Y_{l_{3} m_{3}}(\hat{x}) \\
& \times \int_{0}^{\infty} x^{2} d x j_{l_{1}}\left(k_{1} x\right) j_{l_{2}}\left(k_{2} x\right) j_{l_{3}}\left(k_{3} x\right)\left(C_{l_{1}}^{-1} C_{l_{2}}^{-1} C_{l_{3}}^{-1}\right)_{\mathrm{obs}} \\
& \times\left(\frac{2}{\pi}\right)^{3} \int d k_{1} d k_{2} d k_{3}\left(k_{1} k_{2} k_{3}\right)^{2} B\left(k_{1}, k_{2}, k_{3}\right) \Delta\left(k_{1}\right) \\
& \times \Delta\left(k_{2}\right) \Delta\left(k_{3}\right) j_{l_{1}}\left(k_{1} R_{D}\right) j_{l_{2}}\left(k_{2} R_{D}\right) j_{l_{3}}\left(k_{3} R_{D}\right) \\
& \times\left(\alpha_{l_{1} m_{1}} \alpha_{l_{2} m_{2}} \alpha_{l_{3} m_{3}}\right)_{\mathrm{obs}},
\end{aligned}
$$

where $B\left(k_{1}, k_{2}, k_{3}\right)$ is the primordial bispectrum obtained from the three-point function, as defined in Eq. (17). In this manner, the sought constraints are obtained. The best results, corresponding to the so-called, local, equilateral, and orthogonal shape of non-Gaussianities using the WMAP 7 year data [47], yield $f_{\mathrm{NL}}^{\text {local }}=32 \pm 21(1 \sigma)$, $f_{\mathrm{NL}}^{\text {equil }}=26 \pm 140(1 \sigma)$, and $f_{\mathrm{NL}}^{\text {orthog }}=-202 \pm 104(1 \sigma)$. 


\section{THE COLLAPSE MODEL ACCOUNT FOR THE INFLATIONARY ORIGIN OF COSMIC STRUCTURE}

Before proceeding, it seems worthwhile to briefly explain the view we take regarding quantum physics and Einstein's gravity. The framework we adopt is based on a description of the problem that allows, at the same time, the quantum treatment of other fields and a classical treatment of gravitation, that is, the realm of semiclassical gravity, together with quantum field theory in curved space-time. We will assume that to be a valid approximation most of the time, with the exception associated precisely with the dynamical collapse, as we will explain below. Such a description of gravitation in interaction with quantum fields is characterized by the semiclassical Einstein equation: $R_{\mu \nu}-(1 / 2) g_{\mu \nu} R=8 \pi G\left\langle\hat{T}_{\mu \nu}\right\rangle$, whereas the other fields, including the inflaton, are treated in the standard quantum field theory fashion. It seems clear that this approximated description would break down in association with the quantum mechanical collapses or state jumps, which we are considering to be part of the underlying quantum theory containing gravitation. The reason for this breakdown is simply that the left-hand side of the equation above has zero divergence $\left(\nabla_{\mu} G^{\mu \nu}=0\right)$, while the divergence of the right-hand side, $\nabla_{\nu}\left\langle\hat{T}^{\mu \nu}\right\rangle$, will be nonvanishing (even discontinuous) in connection with the jumps of the quantum state (such a jump is how we are describing here the self-induced collapse of the wave function).

In this setting, we start from the assumption that, in accordance with the standard inflationary accounts, and as mentioned before, the state of the Universe before the time at which the seeds of structure emerge is described by the H\&I Bunch-Davies vacuum state for the matter degrees of freedom (DOF) and the corresponding H\&I classical Robertson-Walker space-time.

Then, we assume that, at a later stage, the quantum state of the matter fields reaches a stage whereby the corresponding state for the gravitational DOF is forbidden, and a quantum collapse of the matter field wave function is triggered by some unknown physical mechanism. In this manner, the state resulting from the collapse of the quantum state of the matter fields does not need to share the symmetries of the initial state. After the collapse, the gravitational DOF are assumed to be, once more, accurately described by Einstein's semiclassical equation. However, as $\left\langle\hat{T}_{\mu \nu}\right\rangle$ for the new state does not need to have the symmetries of the precollapse state, we are led to a geometry that, generically, will no longer be homogeneous and isotropic.

The starting point of the specific analysis is the same as the standard picture, i.e., the action of a scalar field coupled to gravity:
$S=\int d^{4} x \sqrt{-g}\left[\frac{1}{16 \pi G} R[g]-1 / 2 \nabla_{a} \phi \nabla_{b} \phi g^{a b}-V(\phi)\right]$,

where $\phi$ stands for the inflaton and $V$ stands for the inflaton's potential. One then splits both metric and scalar fields into a spatially homogeneous part ("background") and an inhomogeneous part ("fluctuation"), i.e., $g=g_{0}+$ $\delta g, \phi=\phi_{0}+\delta \phi$.

The background is taken to be the spatially flat Friedmann-Robertson universe with line element $d s^{2}=$ $a(\eta)^{2}\left[-d \eta^{2}+\delta_{i j} d x^{i} d x^{j}\right]$ and the homogeneous scalar field $\phi_{0}(\eta)$. The evolution equations for this background are scalar field equations,

$$
\begin{aligned}
& \ddot{\phi}_{0}+2 \frac{\dot{a}}{a} \dot{\phi}_{0}+a^{2} \partial_{\phi} V[\phi]=0, \\
& 3 \frac{\dot{a}^{2}}{a^{2}}=4 \pi G\left(\dot{\phi}_{0}^{2}+2 a^{2} V\left[\phi_{0}\right]\right) .
\end{aligned}
$$

The scale factor corresponding to the inflationary regime, written in terms of the conformal time, is: $a(\eta)=$ $-1 /\left[H_{I}^{2}(1-\epsilon) \eta\right]$ with $H_{I}^{2} \simeq(8 \pi / 3) G V$. The slow roll parameter $\epsilon \equiv 1-\mathcal{H} / \mathcal{H}^{2}$ is considered to be very small $\epsilon \ll 1$ during the inflationary stage. The Hubble factor $H_{I}$ is approximately constant, and the scalar $\phi_{0}$ field is in the slow roll regime, i.e., $\dot{\phi}_{0}=-\left(a^{3} / 3 \dot{a}\right) V^{\prime}$. According to the standard inflationary scenario, this era is followed by a reheating period in which the Universe is repopulated with ordinary matter fields, a regime that then evolves toward a standard hot big bang cosmology regime leading up to the present cosmological time. The functional form of $a(\eta)$ during these latter periods changes, but we will ignore those details because most of the change in the value of $a$ occurs during the inflationary regime. We will set $a=1$ at the "present cosmological time" and assume that the inflationary regime ends at a value of $\eta=\eta_{0}$, negative and very small in absolute terms $\left(\eta_{0} \simeq\right.$ $-10^{-22} \mathrm{Mpc}$ ).

Next, we turn to consider the perturbations. We shall focus in this work on the scalar perturbations and ignore, for simplicity, the tensor perturbations or gravitational waves. Working in the so-called longitudinal gauge, the perturbed metric is written as

$d s^{2}=a(\eta)^{2}\left[-(1+2 \Psi) d \eta^{2}+(1-2 \Psi) \delta_{i j} d x^{i} d x^{j}\right]$,

where $\Psi$ stands for the scalar perturbation usually known as the Newtonian potential.

The perturbation of the scalar field is related to a perturbation of the energy-momentum tensor and reflected into Einstein's equations, which, at the lowest order, lead to the following constraint equation for the Newtonian potential:

$$
\nabla^{2} \Psi=4 \pi G \dot{\phi}_{0} \delta \dot{\phi}=s \delta \dot{\phi},
$$

where we introduced the abbreviation $s \equiv 4 \pi G \dot{\phi}_{0}$. 
Now, we consider in some detail the quantum theory of the field $\delta \phi$. It is convenient to work with the rescaled field variable $y=a \delta \phi$ and its conjugate momentum $\pi=\dot{y}-y \dot{a} / a$. For simplicity, we set the problem in a finite box of side $L$, which can be taken to $\infty$ at the end of all calculations. We decompose the field and momentum operators as

$$
\begin{aligned}
\hat{y}(\eta, \vec{x}) & =\frac{1}{L^{3}} \sum_{\vec{k}} e^{i \vec{k} \cdot \vec{x}} \hat{y}_{\vec{k}}(\eta), \\
\hat{\pi}(\eta, \vec{x}) & =\frac{1}{L^{3}} \sum_{\vec{k}} e^{i \vec{k} \cdot \vec{x}} \hat{\pi}_{\vec{k}}(\eta),
\end{aligned}
$$

where the sum is over the wave vectors $\vec{k}$ satisfying $k_{i} L=2 \pi n_{i}$ for $i=1,2,3$, with $n_{i}$ integer, and where $\hat{y}_{\vec{k}}(\eta) \equiv y_{k}(\eta) \hat{a}_{\vec{k}}+y_{k}^{*}(\eta) \hat{a}_{-\vec{k}}^{\dagger}$ and $\hat{\pi}_{\vec{k}}(\eta) \equiv g_{k}(\eta) \hat{a}_{\vec{k}}+$ $g_{k}^{*}(\eta) \hat{a}_{-\vec{k}}^{\dagger}$ with the usual choice of modes:

$$
\begin{aligned}
& y_{k}(\eta)=\frac{1}{\sqrt{2 k}}\left(1-\frac{i}{\eta k}\right) \exp (-i k \eta), \\
& g_{k}(\eta)=-i \sqrt{\frac{k}{2}} \exp (-i k \eta),
\end{aligned}
$$

which leads to what is known as the Bunch-Davies vacuum.

Note that, according to the point of view we discussed at the beginning of this section and having, at this point, the quantum theory for the relevant matter fields, the effects of the quantum fields on the geometrical variables are codified in the semiclassical Einstein equations. Thus, Eq. (36) must be replaced by

$$
\nabla^{2} \Psi=4 \pi G \dot{\phi}_{0} \delta \dot{\phi}=s\langle\delta \dot{\phi}\rangle=(s / a)\langle\hat{\pi}\rangle .
$$

At this point, one can clearly observe that, if the state of the quantum field is in the vacuum state, the metric perturbations vanish, and thus the space-time is homogeneous and isotropic.

As already mentioned, our proposal is based on the consideration of a self-induced collapse, which we take to operate in close analogy with a "measurement" (but, evidently, with no external measuring apparatus or observer involved). This leads us to want to work with Hermitian operators, which, in ordinary quantum mechanics, are the ones susceptible to direct measurement. Therefore, we must separate both $\hat{y}_{\vec{k}}(\eta)$ and $\hat{\pi}_{\vec{k}}(\eta)$ into their real and imaginary parts $\hat{y}_{\vec{k}}(\eta)=\hat{y}_{\vec{k}}^{R}(\eta)+i \hat{y}_{\vec{k}}{ }^{I}(\eta)$ and $\hat{\pi}_{\vec{k}}(\eta)=\hat{\pi}_{\vec{k}}^{R}(\eta)+i \hat{\pi}_{\vec{k}}^{I}(\eta)$ so that the operators $\hat{y}_{\vec{k}}^{R, I}(\eta)$ and $\hat{\pi}_{\vec{k}}^{R, I}(\eta)$ are Hermitian operators.

So far, we have proceeded in a manner similar to the standard one, except in that we are treating at the quantum level only the scalar field and not the metric fluctuation. At this point, it is worthwhile to emphasize that the vacuum state defined by $\hat{a}_{\vec{k}}, I|0\rangle=0$ is $100 \%$ translational and rotationally invariant (see Appendix A).

For the next step, we must specify in more detail the modeling of the collapse. Then, we must take into account that, after the collapse has taken place, one should consider the continuing evolution of the expectation values of the field variables until the end of inflation and eventually up to the hypersurface of decoupling. In fact, if we wanted to actually compare our analysis directly with observations, we would need evolve the perturbations both through the reheating period and through the decoupling era. This, however, is normally taken into account through the use of appropriate transfer functions, and we will assume that the same procedure could be implemented in the context of the present analysis, but we will not consider it further in the present manuscript.

We will further assume that the collapse is somehow analogous to an imprecise measurement ${ }^{16}$ of the operators $\hat{y}_{\vec{k}}^{R, I}(\eta)$ and $\hat{\pi}_{\vec{k}}^{R, I}(\eta)$. Now, we will specify the rules according to which collapse happens. Again, at this point, our criteria will be simplicity and naturalness. What we have to describe is the state $|\Theta\rangle$ after the collapse.

It seems natural to assume (taking the view that a collapse effect on a state is analogous to some sort of approximate measurement) that after the collapse, the expectation values of the field and momentum operators in each mode will be related to the uncertainties of the precollapse state (recall that the expectation values in the vacuum state are zero). In the vacuum state, $\hat{y}_{\vec{k}}$ and $\hat{\pi}_{\vec{k}}$ individually are distributed according to Gaussian wave functions centered at 0 with spread $\left(\Delta \hat{y}_{\vec{k}}\right)_{0}^{2}$ and $\left(\Delta \hat{\pi}_{\vec{k}}\right)_{0}^{2}$, respectively.

We might consider various possibilities for the detailed form of this collapse. Thus, for their generic form, associated with the ideas above, we write

$$
\left\langle\hat{y}_{\vec{k}}^{R, I}\left(\eta_{k}^{c}\right)\right\rangle_{\Theta}=\lambda_{1} x_{\vec{k}, 1}^{R, I} \sqrt{\left(\Delta \hat{y}_{\vec{k}}^{R, I}\right)_{0}^{2}}=\lambda_{1} x_{\vec{k}, 1}^{R, I}\left|y_{k}\left(\eta_{k}^{c}\right)\right| \sqrt{\hbar L^{3} / 2},
$$

$$
\left\langle\hat{\pi}_{\vec{k}}^{R, I}\left(\eta_{k}^{c}\right)\right\rangle_{\Theta}=\lambda_{2} x_{\vec{k}, 2}^{R, I} \sqrt{\left(\Delta \hat{\pi}_{\vec{k}}^{R, I}\right)_{0}^{2}},=\lambda_{2} x_{\vec{k}, 2}^{R, I}\left|g_{k}\left(\eta_{k}^{c}\right)\right| \sqrt{\hbar L^{3} / 2},
$$

where $x_{\vec{k}, 1}^{R, I}, x_{\vec{k}, 2}^{R, I}$ have been assumed, in our previous works, to be selected randomly from within a Gaussian distribution centered at zero with spread one, and $\eta_{\vec{k}}^{c}$ represents the

\footnotetext{
${ }^{16}$ An imprecise measurement of an observable is one in which one does not end up with an exact eigenstate of that observable but rather with a state that is only peaked around the eigenvalue. Thus, we could consider measuring a certain particle's position and momentum so as to end up with a state that is a wave packet with both position and momentum defined to a limited extent and which, of course, does not entail a conflict with Heisenberg's uncertainty bound.
} 
time of collapse for each mode. Here, $\lambda_{1}$ and $\lambda_{2}$ are parameters taking the values 0 or 1 that allow us to specify the kind of collapse proposal we want to consider. (The main ones we have considered in Refs. [4,8,9] are $\lambda_{1}=$ $\lambda_{2}=1$ for the symmetric collapse and $\lambda_{1}=0, \lambda_{2}=1$ for the Newtonian collapse). At this point, we must emphasize that our Universe corresponds to a single realization of these random variables, and, thus, each of these quantities $x_{\vec{k}, 1}^{R, I}, x_{\vec{k}, 2}^{R, I}$ has a single specific value. The fact that we can represent the specific details of the first inhomogeneities and anisotropies, the seeds of cosmic structure, is something that has no counterpart on the standard treatments. It is clear that one can now investigate how the different specific proposals for the process of collapse could affect the statistics of the $x_{\vec{k}, 1}^{R, I}, x_{\vec{k}, 2}^{R, I}$. One could now inquire about both, the statistics of these quantities in some imaginary ensemble of possible universes as well as the statistics of such quantities for the particular Universe we inhabit.

Regarding the collapse models, it should be clear that there are many other possibilities that we have not even thought about and that might require drastically modified formalisms. In fact, in a recent work [13], grounds were found that suggest a correlation between the $x_{\vec{k}, 1}^{R, I}, x_{\vec{k}, 2}^{R, I}$ of any mode with those of their higher harmonics (something reminiscent of the so-called parametric resonances found in quantum optics in materials with nonlinear response functions [48]). As we noted in Ref. [14] that particular types of correlations, in turn, would lead to a very specific signature, which might be looked for in the statistical features of the CMB.

Returning to the specific models we have described above, we need to compute the relevant expectation values of the field operators in the post-collapse state $|\Theta\rangle$ at the relevant times. For each specific model, we do this by using Eqs. (40) and (41) above and the evolution equations for the expectation values (i.e., using Ehrenfest's theorem). Thus, one obtains $\left\langle\hat{y}_{\vec{k}}^{R, I}(\eta)\right\rangle$ and $\left\langle\hat{\pi}_{\vec{k}}^{R, I}(\eta)\right\rangle$ for the state that resulted from the collapse, for all later times. The explicit expressions for the $\left\langle\hat{y}_{\vec{k}}^{R, I}(\eta)\right\rangle_{\Theta}$ and $\left\langle\hat{\pi}_{\vec{k}}^{R, I}(\eta)\right\rangle_{\Theta}$ are

$$
\begin{aligned}
\left\langle\hat{y}_{\vec{k}}^{R, I}(\eta)\right\rangle_{\Theta}= & {\left[\frac{\cos D_{k}}{k}\left(\frac{1}{k \eta}-\frac{1}{z_{k}}\right)+\frac{\sin D_{k}}{k}\left(\frac{1}{k \eta z_{k}}+1\right)\right] } \\
& \times\left\langle\hat{\pi}_{\vec{k}}^{R, I}\left(\eta_{\vec{k}}^{c}\right)\right\rangle_{\Theta}+\left(\cos D_{k}-\frac{\sin D_{k}}{k \eta}\right)\left\langle\hat{y}_{\vec{k}}^{R, I}\left(\eta_{\vec{k}}^{c}\right)\right\rangle_{\Theta},
\end{aligned}
$$

$$
\begin{aligned}
\left\langle\hat{\pi}_{\vec{k}}^{R, I}(\eta)\right\rangle_{\Theta}= & \left(\cos D_{k}+\frac{\sin D_{k}}{z_{k}}\right)\left\langle\hat{\pi}_{\vec{k}}^{R, I}\left(\eta_{\vec{k}}^{c}\right)\right\rangle_{\Theta} \\
& -k \sin D_{k}\left\langle\hat{y}_{\vec{k}}^{R, I}\left(\eta_{\vec{k}}^{c}\right)\right\rangle_{\Theta},
\end{aligned}
$$

where $D_{k} \equiv k \eta-z_{k}$ and $z_{k} \equiv k \eta_{\vec{k}}^{c}$. This calculation is explicitly done in Refs. [4,11].
With this information at hand, we can now compute the perturbations of the metric after the collapse of all the modes. ${ }^{17}$

\section{A. Connection to observations}

Now, we must put together our semiclassical description of the gravitational DOF and the quantum mechanics description of the inflaton field. We recall that this entails the semiclassical version of the perturbed Einstein equation that, in our case, leads to Eq. (39). The Fourier components at the conformal time $\eta$ are given by

$$
\Psi_{\vec{k}}(\eta)=-\sqrt{\frac{\epsilon}{2}} \frac{H_{I} \hbar}{M_{P} k^{2}}\left\langle\hat{\pi}_{\vec{k}}(\eta)\right\rangle,
$$

where we have used the fact that, during inflation, $s=$ $\sqrt{\epsilon / 2}\left(a H_{I} / M_{P}\right)$, with $M_{P}$ as the reduced Planck mass $M_{P}^{2} \equiv \hbar^{2} /(8 \pi G)$. The expectation value depends on the state of the quantum field; therefore, as we already noted, prior to the collapse, we have $\Psi_{\vec{k}}(\eta)=0$, and the spacetime is still homogeneous and isotropic at the corresponding scale. However, after the collapse takes place, the state of the field is a different state with new expectation values that generically will not vanish, indicating that, after this time, the Universe becomes anisotropic and inhomogeneous at the corresponding scale. We now can reconstruct the space-time value of the Newtonian potential using

$$
\Psi(\eta, \vec{x})=\frac{1}{L^{3}} \sum_{\vec{k}} e^{i \vec{k} \cdot \vec{x}} \Psi_{\vec{k}}(\eta),
$$

to extract the quantities of observational interest.

In order to connect with the observations, we shall relate the expression (44) for the evolution of the Newtonian potential during the early phase of accelerated expansion to the small anisotropies observed in the temperature of the cosmic microwave background radiation, $\delta T(\theta, \varphi) / T_{0}$ with $T_{0} \approx 2.725 \mathrm{Kas}$ the temperature average. They are considered the fingerprints of the small perturbations pervading the Universe at the time of decoupling, and undoubtedly any model for the origin of the seeds of cosmic structure should account for them. As already mentioned in Sec. I, these data can be described in terms of the coefficients $\alpha_{l m}$ of the multipolar series expansion, i.e., Eq. (5). The different multipole numbers $l$ correspond to different angular scales: low $l$ to large scales and high $l$ to small scales. At large angular scales $(l \lesssim 20)$, the Sachs-Wolfe effect is the dominant source for the anisotropies in the $\mathrm{CMB}$. That effect relates the anisotropies in the temperature observed today on the celestial sphere to the inhomogeneities in the Newtonian potential on the last scattering surface,

\footnotetext{
${ }^{17}$ In fact, we need only be concerned with the relevant modes, those that affect the observational quantities in a relevant way. Modes that have wavelengths that are either too large or too small are irrelevant in this sense.
} 


$$
\frac{\delta T}{T_{0}}(\theta, \varphi)=\frac{1}{3} \Psi\left(\eta_{D}, \vec{x}_{D}\right) .
$$

Here, $\eta_{D}$ is the conformal time of decoupling that lies in the matter-dominated epoch, and $\vec{x}_{D}=$ $R_{D}(\sin \theta \sin \varphi, \sin \theta \cos \varphi, \cos \theta)$, with $R_{D}$ as the radius of the last scattering surface. Furthermore, using Eq. (45) and $e^{i \vec{k} \cdot \vec{x}_{D}}=4 \pi \sum_{l m} i^{l} j_{l}\left(k R_{D}\right) Y_{l m}(\theta, \varphi) Y_{l m}^{*}(\hat{k})$, the expression (5) for $\alpha_{l m}$ can be rewritten in the form (6). The transfer function $\Delta(k)$ represents the evolution of the Newtonian potential from the end of inflation $\eta_{R}$ to the time of decoupling $\eta_{D}$, i.e., $\Psi_{\vec{k}}\left(\eta_{D}\right)=\Delta(k) \Psi_{\vec{k}}\left(\eta_{R}\right)$.

Substituting Eq. (43) in Eq. (44) and using Eqs. (40) and (41) gives

$$
\begin{aligned}
\Psi_{\vec{k}}\left(\eta_{R}\right)= & \frac{-(L \hbar)^{3 / 2} \sqrt{\epsilon} H_{I}}{2 \sqrt{2} M_{P} k^{3 / 2}}\left[\lambda_{2}\left(\cos D_{k}+\frac{\sin D_{k}}{z_{k}}\right)\left(x_{\vec{k}, 2}^{R}+i x_{\vec{k}, 2}^{I}\right)\right. \\
& \left.-\lambda_{1} \sin D_{k}\left(1+\frac{1}{z_{k}^{2}}\right)^{1 / 2}\left(x_{\vec{k}, 1}^{R}+i x_{\vec{k}, 1}^{I}\right)\right] .
\end{aligned}
$$

Finally, using Eq. (47) in Eq. (6) yields

$$
\begin{aligned}
\alpha_{l m}= & -\frac{\pi i^{l} \hbar^{3 / 2} \sqrt{2 \epsilon} H_{I}}{3(L k)^{3 / 2} M_{P}} \sum_{\vec{k}} \Delta(k) j_{l}\left(k R_{D}\right) Y_{l m}^{*}(\hat{k}) \\
& \times\left[\lambda_{2}\left(\cos D_{k}+\frac{\sin D_{k}}{z_{k}}\right)\left(x_{\vec{k}, 2}^{R}+i x_{\vec{k}, 2}^{I}\right)\right. \\
& \left.-\lambda_{1} \sin D_{k}\left(1+\frac{1}{z_{k}^{2}}\right)^{1 / 2}\left(x_{\vec{k}, 1}^{R}+i x_{\vec{k}, 1}^{I}\right)\right],
\end{aligned}
$$

note that in Eqs. (47) and (48), $D_{k}$ is evaluated at $\eta_{R}$, i.e., $D_{k}\left(\eta_{R}\right)=k \eta_{R}-z_{k}$.

It is worthwhile to mention that the relation of $\alpha_{l m}$ with the Newtonian potential, as obtained in Eq. (48), within the collapse framework has no analogue in the usual treatments of the subject. It provides us with a clear identification of the aspects of the analysis where the "randomness" is located. In this case, it resides in the randomly selected values $x_{\vec{k}, 1}^{\mathrm{R}, \mathrm{I}}, x_{\vec{k}, 2}^{\mathrm{R}, \mathrm{I}}$ that appear in the expressions of the collapses associated with each of the modes. Here, we also find a clarification of how, in spite of the intrinsic randomness, we can make any prediction at all. The individual complex quantities $\alpha_{l m}$ correspond to large sums of complex contributions, each one having a certain randomness but leading in combination [i.e., the sum of contributions appearing in Eq. (48)] to a characteristic value in just the same way as a random walk made of multiple steps. In other words, the justification for the use of statistics in our approach is that the quantity $\alpha_{l m}$ is the sum of contributions from the collection of modes, each contribution being a random number leading to what is, in effect, a sort of "two-dimensional random walk," for which the total displacement corresponds to the observational quantity. Nothing like this can be found in the most popular accounts, in which the issues we have been focussing on are hidden in a maze of often unspecified assumptions and unjustified identifications [5].

Thus, according to Eq. (48), all the modes contribute to $\alpha_{l m}$, with a complex number. If we had the outcomes characterizing each of the individual collapses, we would be able to predict the exact value of each of these individual quantities. However, we have, at this point, no other access to such information than the observational quantities $\alpha_{l m}$ themselves.

We hope to be able to say something about these, but doing so requires the consideration of further hypothesis regarding the statistical aspects of the physics behind the collapse as well as the conditions previous to them.

As is generally the case with random walks, one cannot hope to estimate the direction of the final displacement. However, one might say something about its estimated magnitude. It is for that reason that we will be focusing on estimating the most likely value of the magnitude:

$$
\begin{aligned}
\left|\alpha_{l m}\right|^{2}= & \frac{16 \pi^{2}}{9 L^{6}} \sum_{\vec{k}, \vec{k}^{\prime}} \Delta(k) \Delta\left(k^{\prime}\right) j_{l}\left(k R_{D}\right) j_{l}\left(k^{\prime} R_{D}\right) Y_{l m}^{*}(\hat{k}) Y_{l m}\left(\hat{k}^{\prime}\right) \\
& \times \Psi_{\vec{k}}\left(\eta_{R}\right) \Psi_{\vec{k}^{\prime}}^{*}\left(\eta_{R}\right) .
\end{aligned}
$$

Note, however, that although, in our approach, each of the quantities $\Psi_{\vec{k}}\left(\eta_{R}\right)$ has, in principle, a particular numerical value, the fact that such value is the result of a quantum collapse characterized by random numbers indicates we cannot make a definite prediction for it. We believe that our approach has, among others, the advantage of offering a clear way to express the prediction for the observable quantities, in a manner in which the aspects that are controlled by randomness are clearly identified. This allows, in principle, the consideration in a separate way of each of the hypotheses and identifications one is interested in making. Our inability of predicting the specific values for the quantities $\left|\alpha_{l m}\right|$, characterizing our observations, is then clearly identified and located in the particular random variables introduced in the collapse hypothesis. But, of course, we want to make predictions. So further considerations become necessary, but the point is that these are clearly identifiable. We will see below what these hypotheses are and how they lead to more specific predictions. One of the advantages we have is that one is able, in principle, to consider removing or modifying each one of those hypotheses. In this case, we can make progress, for instance, by making the assumption that we can regard the specific outcomes characterizing our Universe as a typical member of some hypothetical ensemble of universes.

For example, we are interested in estimating the most likely value of the magnitude of $\left|\alpha_{l m}\right|^{2}$ above, and, in such a hypothetical ensemble, we might hope that it comes very close to our single sample. It is worth emphasizing that, for each $l$ and $m$, we have one single complex number characterizing the actual observations (and, thus, the real Universe we inhabit). For a given $l$, for instance, we should avoid 
confusing ensemble averages with averages of such quantities over the $2 l+1$ values of $m$. The other universes in the ensemble are just figments of our imagination, and there is nothing in our theories that would indicate that they are real.

We can simplify things even further by taking the ensemble average $\overline{\left|\alpha_{l m}\right|^{2}}$ (the bar indicates that we are taking the ensemble average) and identifying it with the most likely value of the quantity, and it is needless to say that these two notions are not exactly equal for many types of ensembles. However, let us, for the moment, ignore this issue and assume the identity of those two values and look at the ensemble average of the quantity $\left|\alpha_{l m}\right|^{2}$, which is given by

$$
\overline{\left|\alpha_{l m}\right|^{2}}=\frac{16 \pi^{2}}{9 L^{6}} \overline{\sum_{\vec{k}, \hat{k}^{\prime}} \Delta(k) \Delta\left(k^{\prime}\right) j_{l}\left(k R_{D}\right) j_{l}\left(k^{\prime} R_{D}\right) Y_{l m}^{*}(\hat{k}) Y_{l m}\left(\hat{k}^{\prime}\right) \Psi_{\vec{k}}\left(\eta_{R}\right) \Psi_{\vec{k}^{\prime}}^{*}\left(\eta_{R}\right)} .
$$

One can, for instance, assume that collapsing events are all uncorrelated (Something not always justified, as exemplified in the analysis of Ref. [13]) and then consider estimating the most likely value; thus,

$$
\left|\alpha_{l m}\right|_{\mathrm{ML}}^{2}=\frac{16 \pi^{2}}{9 L^{6}} \sum_{\vec{k}, \vec{k}^{\prime}} \Delta(k) \Delta\left(k^{\prime}\right) j_{l}\left(k R_{D}\right) j_{l}\left(k^{\prime} R_{D}\right) Y_{l m}^{*}(\hat{k}) Y_{l m}\left(\hat{k^{\prime}}\right) \overline{\Psi_{\vec{k}}\left(\eta_{R}\right) \Psi_{\vec{k}^{\prime}}^{*}\left(\eta_{R}\right)}
$$

Under the assumption of the validity of such an approximation and the additional assumption that the random variables $x_{\vec{k}, 1}^{R}, x_{\vec{k}, 1}^{I}, x_{\vec{k}, 2}^{R}, x_{\vec{k}, 2}^{I}$ are all uncorrelated, we obtain that all the information regarding the "self-collapsing" model will be codified in the quantity

$$
\overline{\Psi_{\vec{k}}\left(\eta_{R}\right) \Psi_{\vec{k}^{\prime}}^{*}\left(\eta_{R}\right)} \text {. }
$$

Generally, one expects this term to be proportional to $\delta_{\vec{k} \vec{k}^{\prime}}$, but alternatives cannot be ruled out. In fact, a case in which this assumption is relaxed was explored in Ref. [14]. Furthermore, we will take the limit $-k \eta_{R} \rightarrow 0$ in Eq. (52), which can be expected to be appropriate when restricting interest to the modes that are "outside the horizon" at the end of inflation (including the modes that give a major contribution to the observationally relevant quantities).

Then, with the help of Eq. (47) and after taking the continuum limit $(L \rightarrow \infty)$, we obtain

$$
\left|\alpha_{l m}\right|_{\mathrm{ML}}^{2}=\frac{\hbar^{3} \epsilon H_{I}^{2}}{36 \pi M_{P}^{2}} \int \frac{d k}{k} \Delta^{2}(k) j_{l}^{2}\left(k R_{D}\right) C(k),
$$

where some of the information regarding that a collapse has occurred is contained in the function $C(k){ }^{18}$ The

\footnotetext{
${ }^{18}$ The standard amplitude for the spectrum is usually presented as $\propto V /\left(\epsilon M_{P}^{4}\right) \propto H_{I}^{2} /\left(M_{P}^{2} \epsilon\right)$. The fact that $\epsilon$ is in the denominator leads, in the standard picture, to a constraint scale for $V$. However, in Eq. (53), $\epsilon$ is in the numerator. This is because we have not used (and, in fact, we will not) explicitly the transfer function $\Delta(k)$. In the standard literature, it is common to find the power spectrum for the quantity $\zeta(x)$, a field representing the curvature perturbation in the comoving gauge. This quantity is constant for modes "outside the horizon" (irrespectively of the cosmological epoch); thus, it avoids the use of the transfer function. The quantity $\zeta$ can be defined in terms of the Newtonian potential as $\zeta \equiv$ $\Psi+(2 / 3)\left(\mathcal{H}^{-1} \dot{\Psi}+\Psi\right) /(1+\omega)$, with $\omega \equiv p / \rho$. For largescale modes $\zeta_{\vec{k}} \simeq \Psi_{\vec{k}}\left[(2 / 3)(1+\omega)^{-1}+1\right]$, and during inflation, $1+\omega=(2 / 3) \epsilon$. For these modes, $\zeta_{\vec{k}} \simeq \Psi_{\vec{k}} / \epsilon$, and the power spectrum is $\mathcal{P}_{\zeta}(k)=\mathcal{P}_{\Psi}(k) / \epsilon^{2} \propto H_{I}^{2} /\left(M_{P}^{2} \epsilon\right) \propto V /\left(\epsilon M_{P}^{4}\right)$, which contains the correct amplitude. For a detailed discussion regarding the amplitude within the collapse framework, see Ref. [10].
}

explicit form of $C(k)$ for the class of collapse schemes considered here is

$$
C(k)=\lambda_{1}^{2}\left(1+\frac{1}{z_{k}^{2}}\right) \sin ^{2} z_{k}+\lambda_{2}^{2}\left(\cos z_{k}-\frac{\sin z_{k}}{z_{k}}\right)^{2} .
$$

As we have noted in previous works, this quantity becomes a simple constant if the collapse time happens to follow a particular pattern in which the time of collapse of the mode $\vec{k}$ is given by $\eta_{k}^{c}=Z / k$ with $Z$ as a constant. In fact, the standard answer would correspond to $C(k)=$ constant (which can be thought as an equivalent "nearly scaleinvariant power spectrum"). Thus, the result obtained for the relation between the time of collapse and the mode's frequency, i.e., $\eta_{\vec{k}}^{c} k=$ constant, is a rather strong conclusion that could represent relevant information about whatever the mechanism of collapse is.

It is quite clear that if the time of collapse of each mode does not adjust exactly to the pattern $k \eta_{k}^{c}=Z$, then the collapse schemes under consideration (characterized by the values of $\lambda_{1}, \lambda_{2}$ ), or some other one resulting in a nontrivial function $C(k)$, would lead to different predictions for the exact form of the spectrum, and comparing these predictions with the observations can help us to discriminate between the distinct collapse schemes. An analyses of these issues have been presented in Refs. [8,9].

We end this section by noting that the treatment of the statistical aspects in the collapse proposal is quite different from the standard inflationary paradigm. We will deepen this discussion in the next section. However, at this point, the differences should be evident. In the standard accounts, one is going from quantum correlation functions to classical $n$-point functions averaged over an ensemble of universes; then, one goes to $n$-point correlation functions averaged over different regions of our own Universe, and, finally, one relates this last quantity with the observable $\left|\alpha_{l m}\right|^{2}$. These series of steps are not at all direct, and they involve a lot of subtle issues that the standard picture does not provide in a transparent way. On the other hand, within the collapse approach to the subject, the observable 
$\left|\alpha_{l m}\right|^{2}$ is related to the random variables, $x_{k}$, through a two-dimensional (i.e., the result is the sum of individual complex numbers) random walk. As we mentioned, the value of $\left|\alpha_{l m}\right|^{2}$ corresponds then to the "length" of the random walk. This random walk is associated to a particular realization of a physical quantum process (i.e., the collapse of the inflaton's wave function), and as we have only access to one realization - the random walk corresponding to our own Universe-the most natural assumption (but certainly not the only one) is that the average value of the length of the possible random walks, which corresponds to $\overline{\left|\alpha_{l m}\right|^{2}}$, is equal to the most likely value, i.e., to $\left|\alpha_{l m}\right|_{\mathrm{ML}}^{2}$, and this, in turn, is associated with the $\left|\alpha_{l m}\right|^{2}$ of our observable Universe.

\section{FURTHER STATISTICAL ASPECTS}

The first thing we should now note is that there are several statistical issues at play and that, within our approach, various novel ones emerge. One central aspect is the exact nature of the state previous to all collapses, i.e., the state characterizing the first stages of the inflationary regime, and normally taken to be the Bunch-Davies vacuum. There are various possibilities that might modify the nature of that state: For instance, if the field is not truly a free field, and self-interactions are important, one might find correlations between the various modes of the field. These effects could be manifest, for instance, by nonvanishing values of quantities like $\left\langle 0\left|\hat{y}_{\vec{k}} \hat{y}_{\vec{k}^{\prime}}\right| 0\right\rangle$ (as argued in the case studied in Ref. [14]). However, we should be aware not only of the inherent problems of accessing those statistical signatures associated with the fact that we have at our disposal a single Universe but also that our Universe, including the relevant perturbations, is not characterized by the vacuum state but rather by the state that results after the collapses of all the modes, and it is quite clear that the collapse process itself can be a source of unexpected correlations. These would manifest themselves, for example, in correlations between the values taken by the $x_{\vec{k}} \mathrm{~s}$ appearing in the collapse process and which we have so far assumed were different and independent quantities for each mode.

Moreover, we have to note that the quantities that are more or less directly accessible to observational investigation are not the $\left\langle\Theta\left|\hat{y}_{\vec{k}}\right| \Theta\right\rangle$, and the $n$-point functions, in general, for the post-collapse state, but the various $\alpha_{l m} \mathrm{~s}$, and the latter are related to the former, as can be seen in Eq. (48) in a nontrivial way. In fact, as we saw, each $\alpha_{l m}$ corresponds to a sort of two-dimensional random walk (i.e., a sum of complex quantities), and each of the steps is related to $\left\langle\Theta\left|\hat{y}_{\vec{k}}\right| \Theta\right\rangle$. It is, thus, clear that there might be correlations between the various $\alpha_{l m}$ s simply due to the fact that they arise from different combinations of the same random variables. Of course, we should note that the version of the collapse proposal we have presented here is based on the assumption that the elementary collapse processes were associated with the observables $\hat{y}_{\vec{k}}$ and their conjugate momenta according to Eqs. (40) and (41). It is clearly conceivable that the elementary process might have been associated, instead, with other observables. One simple possibility for those alternative observables is the various options offered by linear combinations of the former.

\section{A. The new outlook on non-Gaussianities}

In this section, we discuss the aspects that need modification in the study of primordial non-Gaussianities, in view of the approach we have been discussing to the origin of the primordial fluctuations.

The first point we should stress is that, from the two aspects of cosmology mentioned in Sec. I, we have seen that we have had to modify the first, namely, the nature of the quantum state, in order to be compatible with the existence, at the fundamental (quantum) level, of the inhomogeneities and anisotropies that are behind the emergence of structure and, thus, of everything-including observers-in our Universe.

In other words, the standard physics of the very early Universe had to be supplemented with the collapse hypothesis in order to fully account for the process that created the primordial seeds for large-scale structure. Otherwise, we could not really identify the process by which the inhomogeneity and anisotropies emerged from the initial vacuum.

As in the standard approach, we take the curvature perturbations $\Psi$ to be the generators of the CMB anisotropy, $\delta T / T$. However, in our approach, the observed fluctuations are determined, not just by the initial vacuum state, which is and remains homogeneous and isotropic, but also by the characteristics of the collapse process, besides, of course, by the effects of the late-time physics.

In this more precise and detailed approach, it is clear that, even if the primordial state can be considered as Gaussian, in the sense that the corresponding $n$-point functions are completely determined by the two-point functions - and, thus, the odd $n$-point functions vanishit might still be possible for the collapse processes to drastically affect and modify this. In other words, there exists, in principle, the possibility that the collapse process itself introduces non-Gaussian characteristics into the state. We will not discuss this possibility here, but only point it out as something to have in mind and a topic for future research.

As we have argued, the quantity of observational interest is not really $\langle 0|\hat{\Psi}(\vec{x}, \eta) \hat{\Psi}(\vec{y}, \eta)| 0\rangle$, as the argument to justify that in the standard approach depends not only on accepting the identification $\langle 0|\hat{\Psi}(\vec{x}, \eta) \hat{\Psi}(\vec{y}, \eta)| 0\rangle=$ $\overline{\Psi(\vec{x}, \eta) \Psi(\vec{y}, \eta)}$, where $\Psi(\vec{x}, \eta)$ is taken to be a classical stochastic field and the overline denotes the average over an ensemble of universes, but also on a series of arguments indicating one can replace the ensemble averages with suitable spatial averages of quantities in our Universe. 
As a matter of fact, a clear example of how a careless approach to the statistics at hand can lead to wrong conclusions is brought by the variance $\overline{\Psi^{2}}$. We mentioned in Sec. III that $\overline{\Psi^{2}}$ diverges generically if one does not introduce an $a d$ hoc cutoff for $k$. Therefore, if we consider the temperature fluctuations in a particular point $\vec{x}_{0}$ of the last scattering surface, and we estimate it in terms of $\left\langle 0\left|\hat{\Psi}^{2}\left(\vec{x}_{0}, \eta\right)\right| 0\right\rangle$, we obtain a divergent quantity. Note that we are not saying that the temperature anisotropy is divergent, but only that $\left\langle 0\left|\hat{\Psi}^{2}(x)\right| 0\right\rangle$, during the inflationary period, is divergent (see Appendix B). This divergence at an early state would invalidate any subsequent analysis based on perturbation theory, which works under the assumption that the metric perturbations are small in every point. However, we know from the observational data that these fluctuations of the mean temperature, in any particular point, are rather small $\sim 10^{-5} \mathrm{~K}$. On the other hand, in the collapse proposal, these issues become much less problematic because the scheme indicates which variable we should focus on: the variables subjected to the collapse are not $\hat{y}(\vec{x}, \eta), \hat{\pi}(\vec{x}, \eta)$ but the field modes $\hat{y}_{\vec{k}}(\eta), \hat{\pi}_{\vec{k}}(\eta)$, i.e., the collapse does not occur in the position space, and an independent collapse is assumed for each mode $\vec{k}$. The quantities of observational interest, namely, the $\left|\alpha_{l m}\right|^{2}$ s, depend on the expectation values $\left\langle\hat{y}_{\vec{k}}(\eta)\right\rangle_{\Theta},\left\langle\hat{\pi}_{\vec{k}}(\eta)\right\rangle_{\Theta}$, in the state $|\Theta\rangle$ after the collapse, and, as we have shown, these can be estimated directly in terms of the values of the random variables.

As we saw in the introduction, if we really took $\Psi(\vec{x}, \eta)$ to be Gaussian and allowed the identification of its $n$-point functions with the observations, we would have to accept that such identification holds, in particular, for the onepoint function, and that would lead us to a clear conflict between theory and observation.

Similarly, one must be careful when considering the identification of the quantum three-point function:

$$
\langle 0|\hat{\Psi}(\vec{x}, \eta) \hat{\Psi}(\vec{y}, \eta) \hat{\Psi}(\vec{z}, \eta)| 0\rangle,
$$

with the average over an ensemble of Universes $\overline{\Psi(\vec{x}, \eta) \Psi(\vec{y}, \eta) \Psi(\vec{z}, \eta)}$, and finally, the identification of the latter with the measured quantities as an indicator of non-Gaussian features in the cosmological perturbations.

As we saw, the bispectrum $\overline{\Psi_{\vec{k}_{1}} \Psi_{\vec{k}_{2}} \Psi_{\vec{k}_{3}}}=(2 \pi)^{3} \delta\left(\vec{k}_{1}+\right.$ $\left.\vec{k}_{2}+\vec{k}_{3}\right) B_{\Psi}\left(k_{1}, k_{2}, k_{3}\right)$ is usually said to represent the lowestorder statistics able to distinguish non-Gaussian from Gaussian perturbations because Gaussianity is identified with the requirement that all statistical information is contained in the two-point functions and, thus, implicitly, with the vanishing of all $n$-point functions with $n$ odd. However, the lowest odd integer is not 3 but 1 , and, as we have already seen, there is a serious issue that arises when considering the one-point function. This, we believe, forces us to question and reconsider some of the standard arguments.

In fact, looking anew at the quantities normally associated with the one-point function, we see that we have at our disposal not only the average quantities $C_{l}$ but also, for every value of $l$ and $m$, the individual quantities $\alpha_{l m}$. Each one of those corresponds, in our approach, to different random walks. It could prove very interesting to study the distribution of the pair of real quantities that constitute the complex number $\alpha_{l m}$ : namely, we can look at the plot of, say, the real and imaginary part of $\alpha_{l m}$, i.e., $\operatorname{Re}\left(\alpha_{l m}\right)$ and $\operatorname{Im}\left(\alpha_{l m}\right)$, for a given value of $l$. This set of $2 l+1$ numbers for each one of the real and imaginary parts can naturally be expected to display a Gaussian shape (which, in turn, would make the distribution of $\left|\alpha_{l m}\right|$ a Rayleigh distribution).

This seems to be a particularly relevant analysis, and we do not know of anything like that which has been studied in the literature. It seems to us that the traditional approach does not naturally lead to the consideration of that issue. Looking at the distribution of the corresponding phases should be equally enlightening. Moreover, as we mentioned in the discussion around the Eq. (8), it would be interesting to evaluate the quantity $\bar{\alpha}_{l}$ defined there and compare the result with any of the natural estimates for its value, particularly, the expected ensemble average of its magnitude.

Another point worth revisiting is that it is usually believed that a large detectable amount of non-Gaussianity can be expected when the initial state of the quantum field is not the preferred Bunch-Davies vacuum state. Nevertheless, in the collapse proposal, the quantum state of the field after the collapse, is $|\Theta\rangle \neq|0\rangle$ (the analysis of a particular characterization of the post-collapse state has been done in Ref. [11]). Therefore, the curvature perturbation responsible for the temperature anisotropies in the $\mathrm{CMB}$ is due to the expectation values $\left\langle\hat{y}_{\vec{k}}\right\rangle_{\Theta}$ and $\left\langle\hat{\pi}_{\vec{k}}\right\rangle_{\Theta}$, which, in principle, could generate detectable non-Gaussianities. These quantities are never considered in the standard accounts, and it is clear that a further exploration of these ideas would be required for a serious assessment of their value.

The other delicate issue related to the statistical aspects of the traditional approach is related to the ergodicity assumption. As we already saw in Sec. III, the CMB bispectrum was defined as the three-point correlator of the $\alpha_{l m}$ through $B_{m_{1} m_{2} m_{3}}^{l_{1} l_{2} l_{3}} \equiv \overline{\alpha_{l_{1} m_{1}} \alpha_{l_{2} m_{2}} \alpha_{l_{3} m_{3}}}$. The standard picture forces us to deal with the issue that the rhs represents an average over an ensemble of universes, while we have but one realization $\left\{\alpha_{l_{1} m_{1}}, \alpha_{l_{2} m_{2}}, \ldots, \alpha_{l_{n} m_{n}}\right\}$. To overcome this issue, the standard approach relies on an ergodicity assumption, which identifies the average value of a certain quantity in a process measured over time with the average value measured over the ensemble.

There are various issues that lead one to be concerned about this assumption and the application to the situation at hand. The first thing we must be aware of is that ergodicity is a property of systems in equilibrium, and it is rather unclear why this should be valid regarding the conditions associated with the inflationary regime.

Next, as already mentioned, the ergodicity assumption is translated, in the case at hand, into the notion that the 
volume average of the fluctuations behaves like the ensemble average; "the universe may contain regions where the fluctuation is atypical, but with high probability most regions contain fluctuations with root-mean-square amplitude close to $\sigma$," and, thus, one argues that the probability distribution on the ensemble translates to a probability distribution on smoothed regions of a determined size within our own Universe [44].

There are at least three issues that arise here:

(i) How do we go from the arguments supporting ergodicity in time averages to the corresponding arguments for spatial averages?

(ii) Regarding the $\mathrm{CMB}$, we, in fact, do not have access to the spatial sections that would allow us to investigate the space averages. We only have access to the particular intersection of our past light come with the $3 \mathrm{D}$ hypersurface of decoupling. That is, to a two-sphere that we see as the source of the CMB photons that reach us today: the surface of last scattering. How do we go from spatial averages to averages over that two-sphere? ${ }^{19}$

(iii) Each one of the quantities of interest, $\alpha_{l m}$, is itself already a weighted average over the CMB twosphere [with the weight function given by the corresponding $\left.Y_{l m}(\theta, \varphi)\right]$. Therefore, what would be the role of a new average over the $m s$ ? Why do we need to perform any additional average? In other words, if one is willing to accept that the ensemble averages should coincide with averages over the two-sphere, why would one not also accept that the weighted averages over the two sphere should coincide with the equally weighted average over ensembles? If we were to accept this, we would conclude that the weighted average (with weight $Y_{l m}$ and fixed $l$ and $m$ ) of $\delta T / T$ over the surface of last scattering for our Universe should coincide with the corresponding weighted average of that quantity over the ensemble of universes, without any further averaging over $m$. The problem is that the latter would be zero, but the former is just $\alpha_{l m}$, which, empirically, is not zero. Thus, there must be something wrong with our arguments and assumptions. One should then consider what it is, and why.

Let us leave that rhetorical question based on a position we are rejecting and consider again the issue of averaging over $m$. It seems clear that what we are dealing with here are orientation averages: The different $\alpha_{l m}$ would mix among themselves if we were to redefine the orientation of the coordinate chart used to describe the CMB two-sphere. Thus, when we look at the averages that are actually performed in connection with the study of the primordial

\footnotetext{
${ }^{19} \mathrm{We}$ note, in relation to this point, that there are intrinsic problems in considering ergodicity of processes within a twosphere as discussed in Ref. [49].
}

spectrum, we see these are indeed orientation averages. For instance, the observational quantity $C_{l}^{\text {obs }}=\frac{1}{2 l+1} \sum_{m}\left|\alpha_{l m}\right|^{2}$ is just the orientation average value of the magnitude of the $\alpha_{l m}$ s for a fixed value of $l$. In the same way, we see that the angle-averaged bispectrum $B_{l_{1}, l_{2}, l_{3}}(25)$ is an orientation average for fixed $l \mathrm{~s}$, and, as for the same reason as the onepoint function, it is quite unclear how to identify orientation averages with ensemble averages. Thus, the statistical analysis would be more transparent if one would focus on the distribution of the quantities $B_{m_{1} m_{2} m_{3}}^{l_{1} l_{2} l_{3}}$.

As we saw, it is customary to take as an estimator for the nonlinearity parameter the quantity $\hat{f}_{\mathrm{NL}}$ defined in Eq. (32). This seems a bit problematic, as it involves a mixture of theoretical and observational quantities. Ideally, one would like to have the two aspects rather well separated. In fact, even within the standard approach, for the case of the two-point functions, we have on one hand the theoretical quantity,

$$
C_{l}^{\text {th }}=\frac{2}{\pi} \int k^{2} P_{\Psi}(k) \Delta^{2}(k) j_{l}^{2}\left(k R_{D}\right) d k,
$$

and on the other hand the observational quantity,

$$
C_{l}^{\mathrm{obs}}=\frac{1}{2 l+1} \sum_{m}\left|\alpha_{l m}\right|^{2}
$$

This independence of the definitions allows one to cleanly compare theory and observation. It, thus, seems that one would want to consider studying the aspects tied to non-Gaussianity using a quantity that can be equally susceptible to theoretical and observational determination. Here, we would like to propose, based on the considerations we have been discussing, the option we present below.

First, motivated by the quantity defined in Eq. (25), let us introduce the definition of the observed bispectrum as the orientation average,

$\mathcal{B}_{l_{1} l_{2} l_{3}}^{\text {obs }} \equiv \sum_{m_{i}}\left(\begin{array}{ccc}l_{1} & l_{2} & l_{3} \\ m_{1} & m_{2} & m_{3}\end{array}\right)\left(\alpha_{l_{1} m_{1}} \alpha_{l_{2} m_{2}} \alpha_{l_{3} m_{3}}\right)_{\mathrm{obs}}$,

and the definition of the normalized observational reduced bispectrum as the quantity

$\tilde{b}_{l_{1} l_{2} l_{3}}^{\text {obs }} \equiv\left[\sqrt{\frac{\left(2 l_{1}+1\right)\left(2 l_{2}+1\right)\left(2 l_{3}+1\right)}{4 \pi}}\left(\begin{array}{lll}l_{1} & l_{2} & l_{3} \\ 0 & 0 & 0\end{array}\right)\right]^{-1} \mathcal{B}_{l_{1} l_{2} l_{3}}^{\text {obs }}$,

and, finally, let us define the magnitude of the bispectral fluctuations as

$$
\begin{aligned}
\mathcal{F}_{l_{1} l_{2} l_{3}}^{\mathrm{obs}} \equiv & \frac{1}{\left(2 l_{1}+1\right)\left(2 l_{2}+1\right)\left(2 l_{3}+1\right)} \sum_{m_{i}} \mid\left(\alpha_{l_{1} m_{1}} \alpha_{l_{2} m_{2}} \alpha_{l_{3} m_{3}}\right)^{\mathrm{obs}} \\
& -\left.G_{l_{1} l_{2} l_{3}}^{m_{1} m_{2} m_{3}} \tilde{b}_{l_{1} l_{2} l_{3}}^{\mathrm{obs}}\right|^{2}
\end{aligned}
$$


One can then compare this pure observational quantity with the corresponding theoretical estimation characterizing a suitable ensemble average, where each element of the ensemble is specified by a concrete choice of the random numbers $x_{\vec{k}}$ that we have used to represent the collapses. That is, one can carry out a Monte Carlo simulation leading to an ensemble of possible CMB skies characterized by possible choices of $x_{\vec{k}} \mathrm{~s}$ and then characterize each one of those in terms of the corresponding value of $\mathcal{F}_{l_{1} l_{2} l_{3}}^{\text {obs }}$. Finally, one would analyze the degree to which our own real sky is generic when characterized in that manner. It seems clear that this kind of theoretical calculation or simulation cannot be carried out in the standard approach, as there is no place there for the concrete randomness (characterized, in our approach, by the numbers $x_{\vec{k}}$, which would be produced in a simulation of our collapse proposal.

Thus, the study of the quantity displayed in Eq. (60) seems to offer an approach to study the issue at hand that indeed has the advantage of allowing a direct comparison between the purely observational quantities, untainted by theoretical models, and the quantities that are purely defined in terms of such theoretical analysis. This, in fact, seems to share some of the spirit of the analyses made in Refs. [30,31], although our proposal provides a clear option to compute the observational and theoretical quantities in complete separation, and that seems not to be available in the former. The reason for this seems easy to understand: The fact that we maintain a clear distinction between ensemble averages and orientation averages avoids the possibility of the confusion associated with the simple observation that the ensemble average of the quantity

$$
\left(\alpha_{l_{1} m_{1}} \alpha_{l_{2} m_{2}} \alpha_{l_{3} m_{3}}\right)^{\text {obs }}-G_{l_{1} l_{2} l_{3}}^{m_{1} m_{2} m_{3}} \tilde{b}_{l_{1} l_{2} l_{3}}^{\text {obs }}
$$

appearing in Eq. (60), vanishes identically.

The detailed analysis of estimators like this will be carried out in future works, but we wanted to present it as an example of the type of studies that could be motivated by our approach to the whole question of the emergence of structure from quantum fluctuations in the inflationary early Universe.

\section{PREDICTIONS AND DISCUSSION}

Focusing on trying to understand the essence of the emergence of inhomogeneous and anisotropic features from a quantum state, that is, homogeneous and isotropic and in the absence of a measurement process, ${ }^{20}$ has led us to consider modifying the standard approach through the incorporation of the collapse hypothesis.

\footnotetext{
${ }^{20}$ In the early Universe, there were no observers or measuring devices, and, in fact, the conditions for their emergence is the result of the breakdown of such symmetries, so it would seem very odd if one takes a view that they are part of the cause of that breakdown.
}

We have seen in previous works that, despite the fact that the motivation for such considerations seems to be purely philosophical and tied to issues like the measurement problem in quantum mechanics, the analysis has led us to expect certain departures that could potentially be of observational significance.

In previous works, we have focused on two main observationally related issues: the shape of the spectrum and the question of tensor modes. We have argued previously that it would be very unlikely that one could find a scheme in which the function $C(k)$ would be exactly a constant and that some dependence on $k$ is likely to remain in any reasonable collapse scheme, simply because we do not expect those collapses to follow exactly the $\eta_{k}^{c}=Z / k$ rule for the time of collapse for each mode. Any remaining dependency of $C(k)$ on $k$ will lead to slight deviations from the standard form of the predicted spectrum. In fact, analyses of this issue have been carried out in Refs. [8,9], confirming these expectations. These have been used to set the first bounds emerging from the CMB observational data on these kinds of theories.

We have also stated, in earlier works, that the most clear prediction of the novel paradigm we have been proposing is the absence of tensor modes, or at least their very strong suppression. The reason for this can be understood by considering the semiclassical version of Einstein's equations and its role in describing the manner in which the inhomogeneities and anisotropies arise in the metric. As we have explained in our approach, the metric is taken to be an effective description of the gravitational DOF, in the classical regime, and not as the fundamental DOF susceptible to be described at the quantum level. It is, thus, the matter degrees of freedom (which in the present context are represented by the inflaton field), the ones that are described quantum mechanically and which, as a result of a fundamental aspect of gravitation at the quantum level, undergo effective quantum collapse (the reader should recall that our point of view is that gravitation at the quantum level will be drastically different from standard quantum theories, and, in particular, it will not involve universal unitary evolution). This collapse of the quantum state of the inflaton field leads to a nontrivial value for $\left\langle\hat{T}_{\mu \nu}\right\rangle$, which then generates the metric fluctuations. The point is that the energy momentum tensor contains linear and quadratic terms in the expectation values of the quantum matter field fluctuations, which are the source terms determining the geometric perturbations. In the case of the scalar perturbations, we have first-order contributions proportional to $\dot{\phi}_{0}\langle\hat{\dot{\phi} \phi}\rangle$, while no similar first-order terms appear as source of the tensor perturbations (i.e., of the gravitational waves). Of course, it is possible that the collapse scheme works at the level of the simultaneously quantized matter and metric fluctuations, as has been presented in Ref. [50], although, as explained there, we would find it much harder to reconcile that with the broad general 
picture that underlies our current understanding of physical theories.

In the present work, we have focused on the modified statistical considerations associated with this novel paradigm. We have argued that the collapse process itself could be the source of non-Gaussian features. We discussed some difficulties associated with the usual identification of measuring quantities with the quantum $n$-point functions and particularly found that extending the standard arguments to the one-point functions lead to disastrous disagreements with observations.

We have shown that our approach provides expressions that have no parallel in the standard formulations and that allow a precise identification of the location of the randomness, as exemplified by our theoretical formula (48) for $\alpha_{l m}$ in terms of the random numbers characterizing the collapses, namely, the quantities $x_{\vec{k} ; 1}^{R}, x_{\vec{k} ; 1}^{I}, x_{\vec{k} ; 2}^{R}, x_{\vec{k} ; 2}^{I}$. This kind of expression facilitates all resulting statistical considerations, and, in particular, it is the basis for the theoretical estimation of the quantity (60).

We have proposed various novel ways to look into the statistical aspects of the problem:

(i) We indicate the importance of exploring the true nature of the one-point function by studying the degree of deviation from zero of the complex quantity $\bar{\alpha}_{l}^{\text {obs }}=\frac{1}{2 l+1} \sum_{l} \alpha_{l m}^{\text {obs }}$ (i.e., expanding and refining the analysis of Ref. [29]).

(ii) We have argued that it is worthwhile to study the specific form of the distribution of the values of the observed quantities $\left|\alpha_{l m}^{\mathrm{obs}}\right|$ for each fixed $l$.

(iii) We have proposed new characterizations of the quantities normally associated with the bispectrum and the quantum three-point functions, which can be computed both in purely theoretically and in a completely observational fashion. This is the quantity defined in Eq. (60).

It is clear that this work represents only the first step in the study of the statistical aspects of the cosmic structure and its generating process during inflation, within the context of the new paradigm that centers on the collapse hypothesis. Much more work remains to be done, but we hope this can become a research avenue of great richness and one that would lead to important insights, with possible implications not only for the generation of structure itself but for the modification of quantum theory, which would underlie the collapse mechanism and which, as has been argued before, might have a deeper origin at the quantum/gravity interface [5,16,17,51].

\section{ACKNOWLEDGMENTS}

The work of G. L. and D. S. is supported by CONACyT Grant No. 101712 and PAPIIT Project No. IN107412-3. G. L. acknowledges financial support by a CONACyT postdoctoral grant. D. S. was supported in part by sabbatical fellowships from CONACyT and DGAPA-UNAM and the hospitality of the IAFE. The work of S.J.L. is supported by PIP N 0152/10 from Consejo Nacional de Investigaciones Científicas y Técnicas, Argentina.

\section{APPENDIX A: THE BUNCH-DAVIES VACUUM IS HOMOGENEOUS AND ISOTROPIC, CORRELATIONS NOT WITHSTANDING}

Theorem.-The Bunch-Davies vacuum state (this, by the way, is also valid for the Minkowski vacuum state) is homogeneous and isotropic.

Proof.-The vacuum state is defined by $\hat{a}_{\vec{k}}|0\rangle=0$. This is supposed to represent the state of the quantum field after a few e-folds of inflation (up to negligible corrections of order $e^{-N}$, with $N$ as the number of $e$-folds), i.e., the exponential expansion of the Universe takes the metric and all fields to a very simple state, which, in particular, is highly symmetric. It is easy to see that the state $|0\rangle$ is H\&I. The generator of spatial translations is $\hat{\vec{P}}=\sum_{\vec{k}} \vec{k} \hat{a} \hat{k}_{\vec{k}}^{\dagger} \hat{a}_{\vec{k}}$. So a translation by $\vec{D}$ leaves the state unchanged, $e^{i \vec{D} \cdot \hat{\vec{P}}}|0\rangle=|0\rangle$, and, thus, the state is homogeneous. One can equally check that it is isotropic considering the behavior of the state under rotations. Q.E.D.

Furthermore, this is clearly not in contradiction with the existence of quantum correlations, as they do not imply a breakdown of the symmetry. This can be easily seen in a Einstein-Podolsky-Rosen setup. Consider a state of two spin- $1 / 2$ particles that result from the decay of a spin 0 particle.

Let us assume that the decay occurring along the $x$ axis (the particles' momenta are $\vec{P}= \pm P \hat{x}$ with $\hat{x}$ the unit vector in the $\vec{x}$ direction). Using the $\vec{z}$ polarization states as a basis for the Hilbert space of each of the spin-1/2 particles, the state of system after the decay is

$$
|\chi\rangle=\frac{1}{\sqrt{2}}\left(|+\rangle^{(1)}|-\rangle^{(2)}+|+\rangle^{(2)}|-\rangle^{(1)}\right) .
$$

The state can be seen to be invariant under rotations around the $x$ axis (simply because it is an eigenstate with zero angular momentum along that axis). It is, nevertheless, straightforward to compute the correlations between the operators $\hat{S}^{(1)}=\hat{\vec{\sigma}}^{(1)} \cdot \hat{\vec{N}}^{(1)}$ and $\hat{S}^{(2)}=\hat{\vec{\sigma}}^{(2)} \cdot \hat{\vec{N}}^{(2)}$ corresponding to the projectors of the spin along the vectors (taken to be on the $y-z$ plane) $\hat{\vec{N}}^{(1)}$ and $\hat{\vec{N}}^{(2)}$, respectively. The result, as is well known from the studies of Bell's inequalities, is proportional to $\cos \theta$, where $\theta$ is the angle between $\hat{\vec{N}}^{(1)}$ and $\hat{\vec{N}}^{(2)}$. Thus, the existence of these correlations is in no conflict, whatsoever, with the rotational invariance of the state $|\chi\rangle$. It seems that the belief that there is something in the correlations that indicates the breakdown of the symmetry is tied to some implicit intuition suggesting that each one of the particles is in a definite state of spin, even before there are any measurements involved. 
We, of course, know that such notions are in strong conflict with both quantum theory and the experimental facts.

\section{APPENDIX B: DIVERGENCE OF $\left\langle 0\left|\hat{\Psi}^{2}(\vec{x}, \boldsymbol{\eta})\right| 0\right\rangle$}

In order to illustrate a common misconception about the quantity $\left\langle 0\left|\hat{\Psi}^{2}(\vec{x}, \eta)\right| 0\right\rangle$, let us consider the following argument: The gravitational potential that gives temperature anisotropy is not $\hat{\Psi}(\vec{k})$ from the primordial era but $\hat{\Psi}(\vec{k}) \Delta(k)$, where $\Delta(k)$ is the transfer function. Since $\Delta(k) \propto \ln (k) / k^{2}$ for large $k,\left\langle 0\left|\hat{\Psi}^{2}\right| 0\right\rangle$ is convergent in the UV regime.

The previous statement is not correct. The transfer functions characterize the physics that is relevant after the end of inflation (i.e., the physics characterizing the behavior of the radiation and particles that emerge as the result of reheating, including, for instance, the famous plasma oscillations). That is why they are called transfer functions. They indicate how the perturbations that were present during inflation (to be exact, at its end point) evolve during the radiation-dominated era into the perturbations that are present at the time of decoupling, which is the relevant one for what we see in the CMB. The transfer functions are, of course, not relevant at all during the inflationary era itself, which is the era we are focusing on (and the one in which we argue the collapse should occur). The issue, related to the divergence of $\left\langle 0\left|\hat{\Psi}^{2}(\vec{x}, \eta)\right| 0\right\rangle$, clearly refers to the inflationary era. The (rhetorical) question we are posing is the following: If we do not take the expectation value of $\hat{\Psi}_{k}$ to be the inflationary prediction for the $\Psi_{k}$, as that would be zero, and we are instead instructed to compute the vacuum expectation value for quantity $\hat{\Psi}_{k} \hat{\Psi}_{k}$ and to use it in order to make our estimates of $\Psi_{k}$, then, why would it be incorrect to compute the vacuum expectation value of $\hat{\Psi}(x) \hat{\Psi}(x)$ and take it as an estimate of the value of $\Psi(x)$ (during the inflationary era)? The issue is that such an estimate would be infinite, and then the whole scheme of perturbation theory on which the treatment is based would be invalid. We would, therefore, not be able to rely on it, either to consider the study of the predictions regarding the radiation-dominated era or the $\mathrm{CMB}$.

\section{APPENDIX C: ON THE INTERPRETATION OF QUANTUM THEORY AND THE COSMOLOGICAL CONTEXT}

Here, we will briefly consider, for the convenience of the reader, some of the most common views we have found among colleagues regarding the interpretation of quantum physics and their implications for its application to the cosmological problem at hand, and, at the same time, we present our basic perspective on such views. A more detailed discussion of these issues has been presented in Ref. [5], and the reader is advised to turn to that reference for a thorough analysis of the alternative postures taken by researchers in the field.
The issue we are facing is, of course, related to the socalled measurement problem in quantum mechanics [52]. Any reasonably complete discussion of this question and the broader one concerning the interpretation of quantum mechanics would require much more space than what can reasonably be accommodated here, so we point the reader to some of the literature [53]. Here, we will merely present our version of the status of the general problem, touching, when appropriate, on the particular instance that concerns us here: the cosmological setting. That issue has not received to much attention from the physics community, with notable exceptions represented by Penrose [17], Hartle [7], and others.

(i) Quantum physics as a theory of statistical physicsA point of view indicating that quantum mechanics acquires meaning only as it is applied to an ensemble of identically prepared systems [54]. Thus according to this view, a single atom, in isolation, is not described by quantum mechanics. We must avoid getting confused by the correct, but simply distracting, argument that atoms in isolation do not exist. The issue is whether, to the extent to which one can neglect its interactions with other systems, quantum mechanics is applicable to the description of a single atom. One might wonder about the meaning of that question, given that we can not say anything about the atom without making it interact with a measuring device. The question is simply whether or not applying the formalism of quantum mechanics to treat the single isolated atom can be expected to yield correct results regarding subsequent measurements. It is sometimes argued that this is a nonsensical question, as these results are always statistical in nature. However, in fact, this statement is not accurate; for instance, if the atom (e.g., of hydrogen) was known to have been prepared in its ground state, the probability of detecting it in some other energy eigentstate is zero. Thus, there must be something empirical in the description of that single atom by its usual quantum mechanical state. The notion that quantum mechanics is not applicable to a single system [55] is, thus, simply incorrect. However, the most important point in relation with the issue that concerns us in this article is that taking a posture like this about quantum physics, would be admitting from the beginning that we would have no justification in employing such a theory in addressing questions concerning the unique Universe. Note that the situation would not be helped if we assumed that there exists, in some sense of the word, an ensemble of universes, as we would, in principle, have access just to one: ours. Advocates of the standard accounts of inflation usually invoke some 
sort of identification of the statistics with an ensemble of universes and the statistics within one single Universe. However, as we have argued throughout this work, it is paramount to avoid confusion between those types of statistical measures as a matter of principle, even if one would later want to argue they might be identified in some special cases. It should be clear that in order to be able to argue for any such identification, one must be in a position to say something about the individual system. In fact, we can imagine considering any individual system whatsoever, say, a cloud of gas, and constructing an ensemble of similar systems by performing say "all possible rotations and translations of the system." It is clear that the resulting ensemble is, by construction, homogeneous and isotropic. Now, can this be used to say anything about the original system? Clearly, it cannot, simply because our starting point was a completely arbitrary system. Thus, if we negate from the start that our theory could say anything about an individual system, there is no way we can apply it to our Universe. Furthermore, going back to the general case, if a quantum state serves only to represent an ensemble, how is each element of the ensemble to be described? Perhaps, it cannot be described at all. Then, how are we supposed to make statistics over the attributes of such systems?

(ii) Quantum physics as a theory of human knowledge-According to this view, the state of a quantum system is not considered as reflecting anything "objective" about the system in question but just provides a characterization of "what we know about the system". 21 This attitude, naturally rises the question of what there is to be known about the system if not something that pertains to the system. Advocates of this point of view often answer in terms of correlations between the system and the measuring devices. This leads us to consider the question of the significance of these correlations. Note that the meaning of the word "correlation" implies some coincidence of certain conditions pertaining to one object, with some other conditions pertaining to a second object. Therefore, if a quantum state describes such a correlation, there must be some meaning to the conditions pertaining to each one of the objects: the quantum state and the system. Are not these, then, the very same conditions that are described by the quantum mechanical state and those that correspond to the object? If the answer is "no", it must mean that there are further

\footnotetext{
${ }^{21}$ One can find statements in this sense in well-known books, for instance, 'quantum theory is not a theory about reality, it is a prescription for making the best possible predictions about the future, if we have certain information about the past" [56]. See also Ref. [57].
}

descriptions of the object that cannot be cast in the quantum mechanical state vector. On the other hand, if the answer is "yes", we would be implying that the state vector says something about the object in itself. Independently of these issues, it seems rather clear that if we follow the above described view, we would have abandoned the possibility to consider questions about the evolution of the Universe in the absence of sapient beings or to consider the emergence, in that Universe, of the conditions that are necessary for the eventual evolution of humans, while making use of our quantum theory.

(iii) Quantum physics as a noncompletable description of the world-Within this class we consider any posture that effectively, if not explicitly, states, "The theory is incomplete, and no complete theory containing it exists or will ever do." Such a view includes any posture indicating we should use quantum mechanics "as we all know how" and supported by the observation that no violation of quantum mechanics has ever been observed. ${ }^{22} \mathrm{At}$ this point we must note that although this is a literally correct statement, the prescription refers, in fact, to the Copenhagen interpretation, which, as discussed above raises severe interpretational issues that become insurmountable once we leave the laboratory and attempt to apply quantum theory to something like the Universe itself.

The fact is that, in situations in which one cannot identify the system and the measuring apparatus, the observables that are to be measured, the entity carrying out those measurements, and the time at which the measurements take place, the theory does not provide any clearly defined scheme specifying how to make the desired predictions. Thus applies, in particular, to the questions pertaining to the early Universe. However, according to such pragmatic approach we should be satisfied with the fact that the predictions have, in fact, been made and that they do seem to agree with observations. The problem is, that in the absence of a well-defined set of rules, it becomes quite unclear whether or not such "predictions" follow or not from the theory without the use of extraneous and ad hoc, but convenient hypothesis suitably introduced in connection to the particular problem at hand. Especially suspicious are, of course, those predictions that are, in fact, retrodictions, and, on this point, we should recall that, long before inflation was invented, Harrison and Zel'dovich [59] had already concluded what should be the form of the primordial spectrum, based on quite general observations about the nature of the large-scale structure of our Universe.

\footnotetext{
${ }^{22}$ In practice, this view is essentially indistinguishable from the so-called for all practical purposes approach to the matter [58].
} 
(iv) Quantum physics as part of a more complete description of the world-Completing the theory would require something that removes the need for invoking any sort of a priori distinct notions of external measurement apparatus, an external observer, etc. One proposal of this kind is provided by D. Bohm's "pilot wave theory" [60], and, in particular, we note a specific proposal to apply such ideas to the cosmological problem at hand $[61,62]$. As we have mentioned, there are other proposals invoking something like the dynamical reduction models proposed by Pearle [23] and/or Ghirardi et al. [18] and the ideas of R. Penrose about the role of gravitation in modifying quantum mechanics in the merging of the two aspects of nature [17] (see also Ref. [63]). In the context of inflationary cosmology, the works (Refs. [4,8-10,14]) are an example in which the issues are faced directly. Those works represent the position we favor, and which is inspired, in part, by the arguments made in Refs. [17,18,20].

(v) Quantum physics as a complete description of the world-Here we refer to any of the postures indicating that quantum mechanics faces no open issues and that, in particular, the measurement problem has been solved. The advocates of this position fall into groups identified with one of the the main currents: those that subscribe to ideas along the so-called "many world interpretation of quantum mechanics," and consider this to be a solution to the measurement problem, and those that consider that this problem has been solved by the various considerations involving "decoherence." We consider that the many world interpretation does very little to ameliorate the measurement problem, as there is a mapping between what in that approach would be called the "splittings of worlds", 23 and what would be called measurements in the Copenhagen interpretation. In fact one can see that almost every issue that can be raised against in the context of the latter has a corresponding one in the many worlds interpretation choice of basis or context in dealing with measurement problems correspond to the selection of basis for the "world splittings," time of such splittings would need to include those that one takes as the

\footnotetext{
${ }^{23}$ It is often claimed that there is no splitting of the worlds in the many worlds interpretation, but the fact of the matter is that, whenever people make use of it, they cannot avoid talking about things such as "our branch," "the realms we perceive," or other notions that implicitly make use of a notion that is essentially just that of a splitting of the world. One can see this in each specific application of the idea, by focusing on the complete description of what one would take as "the relevant state describing our reality" and following it in time backward and forward. In the inflationary situation at hand, this is easily done by focusing on the symmetry of the state describing the quantum fields.
}

"times of measurements," and so forth. In other words, concerning a specific measurement situation, and the corresponding description within the Many Wolds Interpretation, the issues would be the following: When does a world splitting occur? Why, and under what circumstances does it occur? What constitutes a trigger? And, finally, what selects the basis in which such splittings takes place? The ideas based on a decoherence type solution and its shortcomings will be discussed in some detail in Appendix D.

\section{APPENDIX D: SHORTCOMINGS THE USUAL EXPLANATIONS OF THE EMERGENCE OF PRIMORDIAL INHOMOGENEITIES AND ANISOTROPIES}

We offer here a very brief version of the discussion presented in Ref. [5] of why we find the most widely held views on the way of addressing our problem as lacking. In our experience, these are the "decoherence approach" (perhaps supplemented by the many worlds interpretation of quantum theory) and the "consistent or decohering histories approach."

We will, thus, offer some considerations regarding the degree to which these two proposals do, in general, offer a "solution" to the measurement problem and, particularly, of their applicability in the present context. Again, we suggest turning to Ref. [5] for a more exhaustive discussion of all these issues.

\section{Decoherence}

Decoherence is a direct prediction of quantum mechanics, with very important implications in many experimental situations. The central observation is that, in the general experimental setting involving a quantum mechanical system, one should take into account the fact that generally the system becomes entangled with the environment, consisting of degrees of freedom that are not under the control of the experimentalist and which are, moreover, uninteresting from the point of view of what one is interested in measuring. On the other hand many colleagues seem to think that it has implications that go well beyond that and which represents a complete and satisfactory solution of the measurement problem in quantum mechanics. This is not the case, and the interested reader is directed to consult the literature on the matter (see, for instance, Ref. [64]).

Here, we will limit ourselves to indicating the postures that, in this regard, are held by several people that have considered the issue at length, in order to contrast them with the prevalent notions among inflationary cosmologists.

Let us start with the explicit conclusion by A. Neumaier [65]:

\footnotetext{
"Many physicist nowadays think that decoherence provides a fully satisfying answer to the measurement problem. But this is an illusion."
} 
Also worthwhile is the warning by M. Schlosshauer [66] against misinterpretations:

"...note that the formal identification of the reduced density matrix with a mixed state density matrix is easily misinterpreted as implying that the state of the system can be viewed as mixed too.... the total composite system is still described by a superposition, it follows from the rules of quantum mechanics that no individual definite state can be attributed to one of (the parts) of the system..."

and the explicit refutation by E. Joos [67]:

\begin{abstract}
"Does decoherence solve the measurement problem? Clearly not. What decoherence tells us is that certain objects appear classical when observed, but what is an observation? At some stage we still have to apply the usual probability rules of Quantum Theory."
\end{abstract}

Thus, the decoherence ideas, even if taken together with the many worlds interpretation, clearly fail to offer a satisfactory resolution of the matter in general [68], and, in particular, it fails to do so in connection for the situation we face here.

Let us end by noting that even W. Zurek, one of the most well-known researchers in the field of decoherance, states unequivocally that [69]:

\begin{abstract}
"The interpretation based on the ideas of decoherence and ein-selection has not really been spelled out to date in any detail. I have made a few half-hearted attempts in this direction, but, frankly, I was hoping to postpone this task, since the ultimate questions tend to involve such "anthropic" attributes of the "observership" as "perception", "awareness," or "consciousness," which, at present, cannot be modeled with a desirable degree of rigor."
\end{abstract}

The point is that in the context of inflationary cosmology, in which we want to explain the emergence of the seeds of cosmic structure, and, thus, the emergence of the conditions that would eventually create the conditions for the emergence of galaxies, stars, biology, and intelligent life, we cannot even hope to rely on any of those anthropic notions. Thus, decoherence does not represent an adequate solution to the problem at hand.

\section{Consistent Histories}

The general scheme as described in Ref. [70] is based on the consideration, given a quantum state of the system $|\Phi\rangle$, or, more generally, a density matrix $\hat{\rho}$, for the system at time $t_{0}$, of families of histories characterized by a set of projection operators $\left\{\hat{P}_{n}\left(t_{n}\right)\right\}$, each of which is associated with the system possessing a value of certain physical property in a given range at a given time. ${ }^{24}$ That is, each one of the projector operators is associated with a certain range within the spectrum of a given observable. A given family $F$ of such projectors, is called selfconsistent if the resulting histories do not interfere among themselves. In that case, one may consistently assign probabilities to each individual "coarse-grained history" within the family. ${ }^{25}$

The probability assigned to one particular coarsegrained history within a consistent family is given by

$$
\begin{aligned}
P= & \operatorname{Tr}\left(\hat{P}_{n}\left(t_{n}\right) U\left(t_{n}, t_{n-1}\right) \hat{P}_{n-1} U\left(t_{n-1}, t_{n-2}\right) \ldots \ldots \hat{P}_{2} U\left(t_{2}, t_{1}\right)\right. \\
& \left.\times \hat{P}_{1} U\left(t_{1}, t_{0}\right) \hat{\rho} U\left(t_{n}, t_{0}\right)^{\dagger}\right),
\end{aligned}
$$

where $U\left(t, t^{\prime}\right)$ stands for the standard unitary evolution operators connecting the two times. This approach, apparently, has many followers within the cosmology community, even though it has received some strong criticisms in the foundational physics community [71].

The issue is that, although the scheme works fine once one has selected a particular decoherent family $F$, there exist, in principle, an infinitude of other such decoherent families $F^{\prime}$, which are, however, mutually inconsistent, (i.e., there are elements of $F$ and $F^{\prime}$ that do interfere, and, thus, $\{F\} \cup\left\{F^{\prime}\right\}$ is not a decohering set of histories). This problem, which is well known to the advocates of this approach should according to them be addressed by the "single family rule," which indicates one should never consider, simultaneously, more than one family. Moreover, according to this approach, we should never make any logical inferences while considering together two inconstant sets, as they can produce logical contradictions (see, for instance, the example discussed in Sec. 3 of Ref. [72]. As noted in Ref. [73], it is unclear what would justify this rule within a reasonable ontological view of what the theory is describing.

The issue becomes then how should we select a particular family to be that from which the particular history that represents "the actual one" is to be chosen. (It seems very reasonable that the fact that one assigns probabilities within a family indicates that the interpretation must be that one of the histories in that family is "actualized" in our world. Otherwise, one must wonder what these probabilities refer to [i.e., the probabilities assigned according to Eq. (D1) are probabilities of what? (see, however, Ref. [72])]. Let us emphasize once more that we do not want to take the view that

\footnotetext{
${ }^{24}$ In the cosmological setting, one must use a subtler relational time approach [7], in which one of the dynamical variables is used as an effective time parameter. The cosmological scale factor is a popular choice.

${ }^{25}$ The characterization of the histories as coarse-grained is meant to reelect the fact that the projection operators $\hat{P}_{n}\left(t_{n}\right)$ are generically associated with a finite range of eigenvalues rather than a single eigenvalue.
} 
they are probabilities of "observing a certain value of a physical quantity when that quantity is measured" because the whole point of this program is to have an interpretational framework for quantum theory that avoids using concepts like measurements or "observations" in the discussion (otherwise, one might as well have retained the Copenhagen interpretation).

The fundamental problem is that there is in principle, no clear way to single out one specific family without relying on an a priori given set of questions one interested onthose associated with the quantities whose spectral characteristics one chooses to construct the family-and this ambiguity leads to serious interpretational difficulties [73].

In a specific situation, we might be guided in making the "appropriate" choice, by the questions the experimental setup is "asking" (in fact, this has a close analogy with the use of Bohr's rule in a given experiment). Nevertheless the fact remains that, in general, without such an common sense, or practical guidance, there is no well defined procedure indication how to select the family. We must here emphasize that one is not asking how to select a particular history within the family but how to select a particular family of constant histories from within the collection of all possible decoherent families. The problem here is: what justifies considering that the experimental setup corresponds to asking a particular question; this seems to implicitly assume that the measuring apparatus is always in a state of definite value for the measured quantity and never in the superposition of states of that type. This seems very close to what one does in adopting the Copenhagen interpretation.

Returning to our specific problem, of describing the evolution of the very early universe, there is simply no recipe provided by the theory, that would dictate the selection of the appropriate projector operators and, thus, of the appropriate family (if we require a description that does not make use of the fact of our own existence and our own asking of certain questions as part of the input).

Let us see a clear manifestation of this problem in the cosmological situation of interest: Consider the family of projector operators as is done in Ref. [74], and obtain their results, but then note that, alternatively, we might consider the family in what follows. We next define $P_{\mathrm{HI}}$ to be the projector into the intersection of the kernels of the generators of translations and rotations (note that it is the projector onto the space of homogeneous and isotropic states). Let us further define $P_{\text {non }} \equiv I-P_{\mathrm{HI}}$ the orthogonal projector. Take the initial state for the quantum fluctuations (usually called the vacuum) $\left|\Phi_{0}\right\rangle$, and note that it is homogeneous and isotropic.

The next step is to consider an arbitrary collection of values for time $\left\{t_{i}\right\}$ and construct the family associated with that initial state and the two projector operators $P_{\mathrm{HI}}$ and $P_{\text {non }}$ at each of those times. One can easily check that this procedure defines a family of consistent histories, simply because the dynamics (characterized by the operators $U$ ) preserves the symmetries (homogeneity and isotropy).

Consider now the question of what the probability is that (at a given time, characterized in the appropriate relational way) the Universe is isotropic and homogeneous. Evaluating this using the formula (D1) (and starting with the vacuum state), we find that any history containing the orthogonal projector at any time $P_{\text {non }}$ has zero probability, while the history containing only the operators $P_{\mathrm{HI}}$ has probability one. We seem to be led to conclude that, at any time, the Universe is homogeneous and isotropic. It, thus, can have no inhomogeneities or anisotropies at all. We would then have to face not only such a problematic prediction but also the fact that the approach we followed has led us to two conflicting conclusions: this latter one and the one obtained in, say, Ref. [74].

In fact, this problem is similar to those considered in Ref. [71] and that discussed in Sec. 3 of Ref. [72]. The posture advocated in Ref. [72] is that one should accept all the different families and use only the appropriate one in connection with the particular question one is asking in order to make "bets about the future," while at the same time renouncing the idea that there is a single objective reality. As discussed in Ref. [73], such a posture seems unsustainable in addressing the fact that we humans seem to coincide regarding our appreciation of the "world out there."

Apparently, if choosing to accept the consistent histories approach to the quantum theory, in general, one would have to adopt a rather problematic position close to that of posture b) in Appendix C, with the difficulties already mentioned there. It seems that this is not a satisfactory situation regarding something that ought to serve as a fundamental theory and, in particular, to help us deal with the quantum aspects of the early Universe. The interested reader is referred to the literature, particularly, to the works referred to above, for much more extensive discussions on the matter.
[1] A. Guth and S.-Y. Pi, Phys. Rev. D 32, 1899 (1985); S. W. Hawking, Nucl. Phys. B224, 180 (1983); J. J. Halliwell and S. W. Hawking, Phys. Rev. D 31, 1777 (1985).

[2] J. J. Halliwell, Phys. Rev. D 39, 2912 (1989); C. Kiefer, Nucl. Phys. B, Proc. Suppl. 88, 255 (2000); D. Polarski and A. A. Starobinsky, Classical Quantum Gravity 13, 377 (1996); W. H. Zurek, Report No. QC178:S4:1990, 1990, http://www.osti.gov/bridge/product.biblio.jsp?osti_id= 6356333; R. Branderberger H. Feldman, and V.F. Mukhavov, Phys. Rep. 215, 203 (1992); R. Laflamme 
and A. Matacz, Int. J. Mod. Phys. D 02, 171 (1993); M. Castagnino and O. Lombardi, Int. J. Theor. Phys. 42, 1281 (2003); F. C. Lombardo and D. Lopez Nacir, Phys. Rev. D 72, 063506 (2005); J. Martin, Lect. Notes Phys. 669, 199 (2005); A. O. Barvinsky, A. Y. Kamenshchik, C. Kiefer, and I. V. Mishakov, Nucl. Phys. B551, 374 (1999).

[3] S. Weinberg, Cosmology (Oxford University, New York, 2008), p. 593

[4] A. Perez, H. Sahlmann, and D. Sudarsky, Classical Quantum Gravity 23, 2317 (2006).

[5] D. Sudarsky, Int. J. Mod. Phys. D 20, 509 (2011).

[6] P. M. Pearle, arXiv:0710.0567.

[7] J. B. Hartle, in Proceedings of the 23rd Solvay Conference in Physics: The Quantum Structure of Space and Time, Brussels, Belgium, 2005, edited by D. Gross, M. Henneaux, A.S. Hackensack (World Scientific, Singapore, 2007), p. 272; J. B. Hartle, Int. J. Theor. Phys. 45, 1390 (2006); J. B. Hartle, arXiv:gr-qc/ 9701022; J. B. Hartle, Report No. UCSBTH-90-31,C8912-27; M. Gell-Mann and J. B. Hartle, University of California, Santa Barbara, Report No. PRINT-90-0266.

[8] A. De Unanue and D. Sudarsky, Phys. Rev. D 78, 043510 (2008).

[9] S. J. Landau, C. G. Scoccola, and D. Sudarsky, Phys. Rev. D 85, 123001 (2012).

[10] G. León and D. Sudarsky, Classical Quantum Gravity 27, 225017 (2010).

[11] G. León, A. De Unanue, and D. Sudarsky, Classical Quantum Gravity 28, 155010 (2011).

[12] R. M. Wald (private communication).

[13] A. Diez-Tejedor and D. Sudarsky, J. Cosmol. Astropart. Phys. 07 (2012) 045.

[14] G. León and D. Sudarsky, SIGMA 8, 024 (2012).

[15] S. Weinberg, Report No. UTTG-18-11, 2011.

[16] L. Diosi, Phys. Lett. 105A, 199 (1984); 120A, 377 (1987); 40A, 1165 (1989).

[17] R. Penrose, The Emperor's New Mind (Oxford University, New York, 1989), p. 480; R. Penrose, Gen. Relativ. Gravit. 28, 581 (1996).

[18] G. C. Ghirardi, A. Rimini, and T. Weber, Phys. Rev. D 34, 470 (1986).

[19] P. M. Pearle, Phys. Rev. A 39, 2277 (1989).

[20] A. Bassi and G. C. Ghirardi, Phys. Rep. 379, 257 (2003).

[21] W. C. Myrvold, Stud. Hist. Phil. Mod. Phys. 33, 435 (2002); R. Tumulka, Proc. R. Soc. A 462, 1897 (2006).

[22] D. J. Bedingham, Found. Phys. 41, 686 (2011).

[23] P. Pearle, Phys. Rev. D 13, 857 (1976); Int. J. Theor. Phys. 18, 489 (1979); Phys. Rev. D 29, 235 (1984); Phys. Rev. A 39, 2277 (1989).

[24] J. Martin, V. Vennin, and P. Peter, Phys. Rev. D 86, 103524 (2012).

[25] P. Cañate, P. Pearle, and D. Sudarsky, arXiv:1211.3463 [Phys. Rev. D (to be published)].

[26] V.F. Mukhanov, Physical Foundations of Cosmology (Cambridge University Press, Cambridge, England, 2005).

[27] D. H. Lyth and A. R. Liddle, The Primordial Density Perturbation: Cosmology, Inflation and the Origin of Structure (Cambridge University Press, Cambridge, England, 2009).

[28] See, for instance: ND. Mermin, Phys. Today 38, No. 4, 38 (1985); J. S. Bell, Speakable and Unspeakable in Quantum
Mechanics (Cambridge University Press, Cambridge, England, 1988); R. Penrose, The Road to Reality-A complete guide to the laws of the Universe (Jonathan Cape, London, 2004).

[29] C. Armendariz-Picon, J. Cosmol. Astropart. Phys. 03 (2011) 048

[30] A.P.S. Yadav and B. D. Wandelt, Adv. Astron. 2010, 565248 (2010).

[31] M. Liguori, E. Sefusatti, J.R. Fergusson, and E.P.S. Shellard, Adv. Astron. 2010, 980523 (2010).

[32] E. Komatsu, Ph.D. thesis, Tohoku University, 2001.

[33] E. Komatsu, Classical Quantum Gravity 27, 124010 (2010).

[34] N. Bartolo, E. Komatsu, S. Matarrese, and A. Riotto, Phys. Rep. 402, 103 (2004).

[35] T. J. Allen, B. Grinstein, and M. B. Wise, Phys. Lett. B 197, 66 (1987)

[36] E. Komatsu et al., arXiv:0902.4759.

[37] D. Babich, P. Creminelli, and M. Zaldarriaga, J. Cosmol. Astropart. Phys. 08 (2004) 009.

[38] P. Creminelli and M. Zaldarriaga, J. Cosmol. Astropart. Phys. 10 (2004) 006.

[39] L. Senatore, K. M. Smith, and M. Zaldarriaga, J. Cosmol. Astropart. Phys. 01 (2010) 028.

[40] D. S. Salopek and J.R. Bond, Phys. Rev. D 42, 3936 (1990).

[41] D. S. Salopek and J.R. Bond, Phys. Rev. D 43, 1005 (1991).

[42] J. L. Lehners, Adv. Astron. 2010, 903907 (2010).

[43] C. T. Byrnes and K. Y. Choi, Adv. Astron. 2010, 724525 (2010).

[44] D. Seery and J. C. Hidalgo, J. Cosmol. Astropart. Phys. 07 (2006) 008

[45] A. P. S. Yadav, E. Komatsu, B. D. Wandelt, M. Liguori, F. K. Hansen, and S. Matarrese, Astrophys. J. 678, 578 (2008).

[46] P. Creminelli, A. Nicolis, L. Senatore, M. Tegmark, and M. Zaldarriaga, J. Cosmol. Astropart. Phys. 05 (2006) 004

[47] E. Komatsu et al., Astrophys. J., Suppl. Ser. 192, 18 (2011).

[48] M. Fox, Quantum Optics An Introduction, Oxford Master Series in Physics (Oxford University, New York, 2006).

[49] L. P. Grishchuk and J. Martin, Phys. Rev. D 56, 1924 (1997).

[50] A. Diez-Tejedor, G. Leon, and D. Sudarsky, Gen. Relativ. Gravit. 44, 2965 (2012)

[51] D. Sudarsky, Int. J. Mod. Phys. D 20, 821 (2011).

[52] For reviews about the various approaches to the measurement problem in quantum mechanics, see, for instance, the classical reference M. Jammer, Philosophy of Quantum Mechanics. The Interpretations of Quantum Mechanics in Historical Perspective (Wiley, New York, 1974); R. Omnes, The Interpretation of Quantum Mechanics (Princeton University, Princeton, NJ, 1994); and the more specific critiques S.L. Adler, Stud. Hist. Phil. Mod. Phys. 34, 135 (2003); A. Elby, Found. Phys. Lett. 6, 5 (1993).

[53] For a recent discussion on the status of the subject, see, for instance, G. C. Ghirardi, arXiv:0904.0958.

[54] L.E. Ballentine, Rev. Mod. Phys. 42, 358 (1970).

[55] Y. Aharonov and L. Vaidman, Phys. Lett. A 178, 38 (1993). 
[56] A. Peres, Quantum Theory Concepts and Methods (Kluwer Academic Publishers, the Netherlands, 1993).

[57] J. B. Hartle, Am. J. Phys. 36, 704 (1968).

[58] J. S. Bell, Phys. World 3, 33 (1990).

[59] E. R. Harrison, Phys. Rev. D 1, 2726 (1970); Y. B. Zeĺdovich, Mon. Not. R. Astron. Soc. 160, 1 (1972).

[60] The classic reference is Phys. Rev. 85, 166 (1952), although the first proposal was in fact made in by L. de Broglie at in Electrons et Photons: Rapports et Discussions du Cinquieme Conseil de Physique (Gauthier-Villars, Paris, 1928).

[61] A. Valentini, Phys. Rev. D 82, 063513 (2010).

[62] N. Pinto-Neto, G. Santos, and W. Struyve, Phys. Rev. D 85, 083506 (2012).

[63] J. Anandan, Found. Phys. 29, 333 (1999).

[64] S. L. Adler, Stud. Hist. Phil. Mod. Phys. 34, 135 (2003).

[65] A. Neumaier, http://www.mat.univie.ac.at/ neum/ physics-faq.txt.

[66] M. Schlosshauer, Rev. Mod. Phys. 76, 1267 (2005).

[67] E. Joos, in Decoherence Theoretical, Experimental and Conceptual Problems, edited by P. Blanchard, D. Giulini,
E. Joos, C. Kiefer, and I.O. Stamatescu (Springer, New York, 1999).

[68] For further discussion of the issue and other points of view, see, for instance: A. Kent, Int. J. Mod. Phys. A 05, 1745 (1990); T. Sakaguchi, arXiv:gr-qc/9704039; J. Garriga and A. Vilenkin, Phys. Rev. D 64, 043511 (2001); C. Barrabes and V. P. Frolov, Phys. Rev. D 53, 3215 (1996).

[69] W. H. Zurek, Phil. Trans. R. Soc. A 356, 1793 (1998).

[70] R. B. Griffiths, Phys. Rev. Lett. 70, 2201 (1993); R. B. Griffiths, Phys. Rev. A 57, 1604 (1998); R. Omnes, Rev. Mod. Phys. 64, 339 (1992); J. Math. Phys. (N.Y.) 38, 697 (1997); M. Gell-Mann and J. B. Hartle, Phys. Rev. D 47, 3345 (1993).

[71] A. Kent, Phys. Rev. Lett. 78, 2874 (1997); F. Dowker and A. Kent, J. Stat. Phys. 82, 1575 (1996); A. Bassi and G. C. Ghirardi, Phys. Lett. A 257, 247 (1999); A. Bassi and G. C. Ghirardi, Phys. Lett. A 265, 153 (2000).

[72] J. B. Hartle, J. Phys. A: Math. Theor. 40, 3101 (2007).

[73] E. Okon and D. Sudarsky, arXiv:1301.2586.

[74] J. B. Hartle, S. W. Hawking, and T. Hertog, Phys. Rev. D 82, 063510 (2010). 